ARTICLE

\title{
Perturbed structural dynamics underlie inhibition and altered efflux of the multidrug resistance pump AcrB
}

\author{
Eamonn Reading (10) 1,5,6凶 , Zainab Ahdash ${ }^{1,5}$, Chiara Fais², Vito Ricci ${ }^{3}$, Xuan Wang-Kan (10 ${ }^{3}$, Elizabeth Grimsey ${ }^{3}$, \\ Jack Stone ${ }^{3}$, Giuliano Malloci (i) ${ }^{2}$, Andy M. Lau', Heather Findlay' ${ }^{1}$, Albert Konijnenberg${ }^{4}$, Paula J. Booth', \\ Paolo Ruggerone (iD) ${ }^{2}$, Attilio V. Vargiu (i) ${ }^{2}$, Laura J. V. Piddock (i) ${ }^{3}$ \& Argyris Politis (1) ${ }^{1,6 凶}$
}

Resistance-nodulation-division efflux pumps play a key role in inherent and evolved multidrug resistance in bacteria. AcrB, a prototypical member of this protein family, extrudes a wide range of antimicrobial agents out of bacteria. Although high-resolution structures exist for AcrB, its conformational fluctuations and their putative role in function are largely unknown. Here, we determine these structural dynamics in the presence of substrates using hydrogen/deuterium exchange mass spectrometry, complemented by molecular dynamics simulations, and bacterial susceptibility studies. We show that an efflux pump inhibitor potentiates antibiotic activity by restraining drug-binding pocket dynamics, rather than preventing antibiotic binding. We also reveal that a drug-binding pocket substitution discovered within a multidrug resistant clinical isolate modifies the plasticity of the transport pathway, which could explain its altered substrate efflux. Our results provide insight into the molecular mechanism of drug export and inhibition of a major multidrug efflux pump and the directive role of its dynamics.

\footnotetext{
${ }^{1}$ Department of Chemistry, King's College London, Britannia House, 7 Trinity Street, London SE1 1DB, UK. ${ }^{2}$ Department of Physics, University of Cagliari, Cittadella Universitaria, S.P. Monserrato-Sestu, 09042 Monserrato, (CA), Italy. ${ }^{3}$ Antimicrobials Research Group, Institute of Microbiology and Infection, College of Medical and Dental Sciences, The University of Birmingham, Birmingham B15 2TT, UK. ${ }^{4}$ Thermo Fisher Scientific, Zwaanstraat $31 \mathrm{G} / \mathrm{H}, 5651 \mathrm{CA}$ Eindhoven, The Netherlands. ${ }^{5}$ These authors contributed equally: Eamonn Reading, Zainab Ahdash. ${ }^{6}$ These authors jointly supervised this work: Eamonn

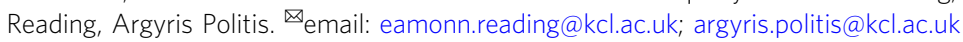


A $\mathrm{crB}$ is a homotrimeric integral membrane protein that forms part of the tripartite AcrAB-TolC efflux pump ${ }^{1}$ (Fig. 1a, b and Supplementary Fig. 1). Energized by the proton-motive force, AcrB transports a broad variety of toxic substances, including antibiotics, outside of the cell through a channel formed by the periplasmic adaptor protein, AcrA, and outer membrane channel, TolC ${ }^{2,3}$. It is constitutively expressed in many pathogenic Gram-negative bacteria and, with its homologues forming the most clinically relevant pumps, has become a target for drug discovery to tackle multidrug resistance (MDR $)^{4-7}$.

Previous structural and biochemical work has enabled drug-binding pockets and efflux pathways for AcrB to be proposed $1,2,7-17$. Drug transport is purported to occur via cooperative rotation between three distinct monomer conformations: loose $(\mathrm{L})$, tight $(\mathrm{T})$, and open $(\mathrm{O})$ (Fig. 1c). Where, in the L-state, drugs gain access to the proximal binding pocket (PBP) through entrance channels ${ }^{1,8,18}$. Upon a conformational change to the Tstate, the drug is then moved towards the distal binding pocket (DBP) before being transported through the exit channel of the periplasmic domain, following a second conformational change from the T- to O-state ${ }^{19,20}$. A switch-loop $\left({ }^{615} \mathrm{FGFAGR}^{620}\right)$ acts to separate the PBP from the DBP (Fig. 1b); the conformational flexibility of this region is proposed to be essential for the regulation of substrate binding and export ${ }^{21,22}$.

Each AcrB monomer contains a connecting-loop, which protrudes into the Funnel Domain (DC and DN subdomains) of the a

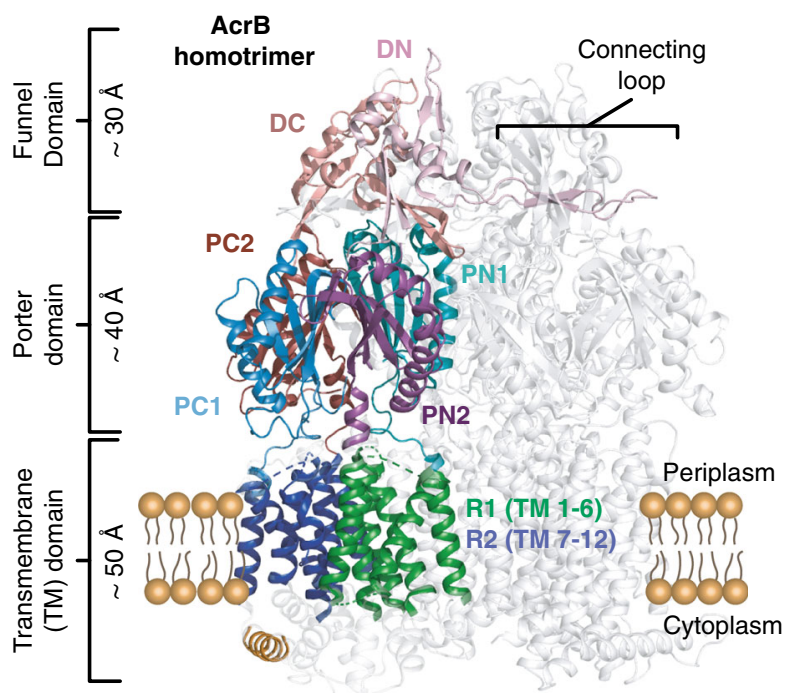

adjacent monomer (Fig. 1a) and has been shown to be important for stabilizing the trimer during the functional rotation ${ }^{23}$. During rotation, the transmembrane (TM) region-which consists of the R1 (TM1-6) and R2 (TM7-12) domains connected by the I $\alpha$-helix $\left({ }^{520}\right.$ FEKSTHHYTDSVGGIL ${ }^{535}$ ) (Fig. 1a, b) -is also postulated to move considerably 14,24

Despite the availability of high-resolution structures of AcrB, it has remained challenging to dissect how its molecular mechanisms are regulated by naturally occurring MDR mutations, efflux pump inhibition and their synergy with antibiotics. One fundamental aspect of its structure that remains unresolved concerns the role of its structural dynamics, which are often crucial for protein function ${ }^{25-28}$. Here, we use hydrogen/deuterium exchange mass spectrometry (HDX-MS) to directly measure changes in AcrB structural dynamics owing to binding of the ciprofloxacin (CIP) antibiotic and the well-studied phenylalanine-arginine- $\beta$ naphthylamide $(\mathrm{PA} \beta \mathrm{N})$ efflux pump inhibitor $(\mathrm{EPI})^{5,7,15,29}$. Investigating both wildtype $\mathrm{AcrB}\left(\mathrm{AcrB}{ }^{\mathrm{WT}}\right)$ and a recently discovered $\mathrm{G} 288 \mathrm{D}$ mutation $\left(\mathrm{AcrB} \mathrm{B}^{\mathrm{G} 288 \mathrm{D}}\right)$, uncovered in a posttherapy MDR clinical isolate of Salmonella Typhimurium ${ }^{30}$, we were able to further understand the structural and functional consequences of substrate and inhibitor binding and clinically relevant mutation.

In this work, we reveal that the $\mathrm{PA} \beta \mathrm{N}$ EPI restricts the intrinsic motions of the drug-binding pockets as part of its mechanism of action and is effective against both AcrBWT and AcrB ${ }^{\mathrm{G} 288 \mathrm{D}}$. We also demonstrate that an EPI can dually bind

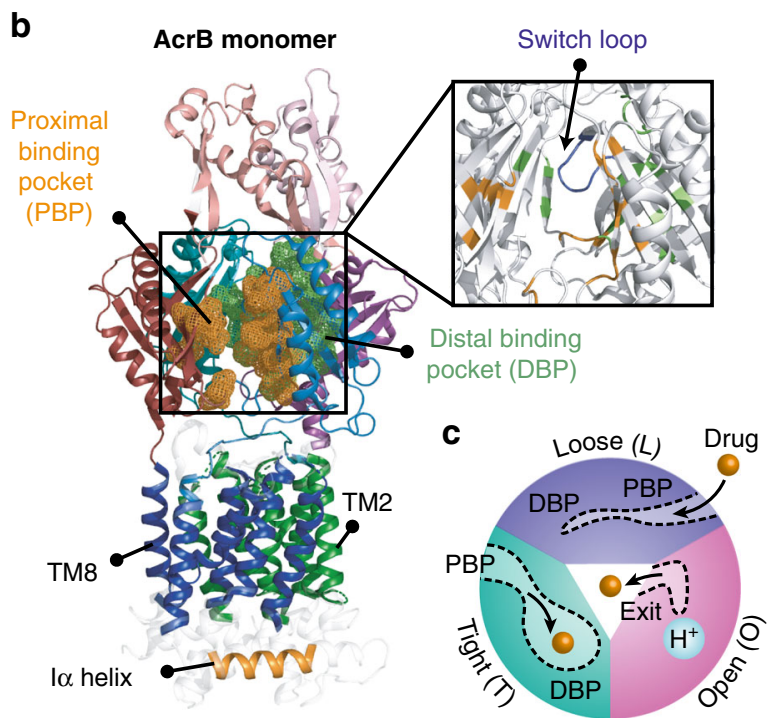

d $H / D$ eXchange $\left(+D_{2} O\right)$
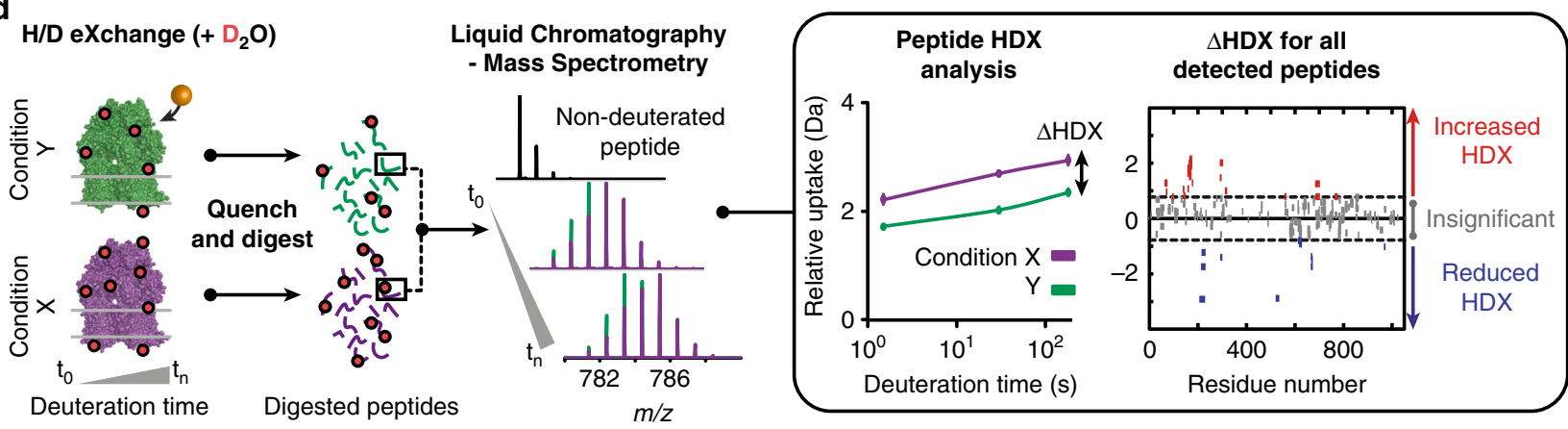

Fig. 1 Summary of structure, function, and HDX-MS of AcrB. a Structure and subdomains of AcrB (PDB:2HRT). b Highlighted regions of functional interest on $A c r B$ monomeric unit. c Functional rotation drug efflux mechanism between the three distinct monomer conformations within trimeric AcrB. d HDX-MS workflow. 
to AcrB alongside an antibiotic, without affecting its inhibitory action. We discover that an MDR mutation in $a c r B$ impacts upon the structural dynamics of the efflux translocation pathway, likely contributing to its modified substrate efflux. Structural dynamics therefore have a critical role in the inhibition and substrate efflux of AcrB. Understanding the effect of these dynamics on structure and function is critical for successful assessment of resistance-nodulation-division (RND) efflux pump mechanism and inhibition, especially in relation to MDR-conferring mutations.

\section{Results}

HDX-MS of AcrB. HDX-MS is a solution-based method which can provide molecular-level information on local protein structure, stability, and dynamics ${ }^{31-33}$. HDX occurs when backbone amide hydrogens are made accessible to $\mathrm{D}_{2} \mathrm{O}$ solvent through structure unfolding and $\mathrm{H}$-bond breakage; HDX is fast within unfolded regions and slow within stably folded regions (i.e. $\alpha$ helices, $\beta$-sheet interiors), where local structural fluctuations which expose an otherwise protected amide hydrogen to solvent transiently are required for HDX to occur. In order to decipher the effect of drug binding and mutation on AcrB structural dynamics, we performed differential HDX $(\triangle \mathrm{HDX})$ analysis between two conditions (e.g. drug-bound and drug-free), which is a sensitive approach for detecting associated structural perturbations between two different protein states (Fig. 1d) $)^{33-35}$.

We optimized HDX-MS conditions on Escherichia coli (E. coli) AcrB solubilized within $n$-Dodecyl- $\beta$-D-Maltopyranoside (DDM) detergent micelles achieving $72 \%$ peptide coverage (Supplementary Fig. 2). A relative fractional deuterium uptake analysis of AcrB revealed that many of its residues form part of stable structures, inferred from the longest labelling time points (0.5-1 h) required for substantial deuterium incorporation ${ }^{32}$ (Supplementary Figs. 3, 4). Its most structurally dynamic regions being discovered within the subdomains of the Porter Domain (PC1, PC2, PN1, and PN2) which, notably, host the main drug-binding pockets (Fig. 1a, b and Supplementary Table 1). Lack of extensive HDX was observed within the TM domains, likely afforded by their protection within the hydrophobic environment of the detergent micelle.

EPI restricts drug-binding pocket dynamics. First, we examined how CIP and PAßN binding affects AcrB ${ }^{\mathrm{WT}}$ structural dynamics. CIP is a licensed antibiotic in clinical use and has been demonstrated to bind to the DBP, PC1/PC2 cleft and central cavity by MD simulations and X-ray crystallography ${ }^{15,29}$. While PA $\beta N$, an EPI and substrate of AcrB, has been shown to bind to similar areas of AcrB as many antibiotics, this binding may be at distinct sites $^{5,15,29}$.

In the presence of CIP only a few regions within the PN2 subdomain, central cavity, R2 domain (TM 7-12) and Iahelix demonstrated significant differential HDX (Fig. 2a, b), suggesting that CIP binding only subtly alters the structural motions of $A c r B$ WT. The presence of $\mathrm{PA} \beta \mathrm{N}$ gave comparably increased HDX within the PN2 subdomain. However, in stark contrast to CIP, inhibitor binding led to $\mathrm{HDX}$ reduction throughout extensive parts of the PC1/PC2 cleft of the drugbinding pockets and within the connecting-loop (Fig. 2a, b and Supplementary Fig. 5). This could signify inhibitor-induced structural stabilization of the drug-binding pocket entrances.

HDX of the I $\alpha$-helix did not significantly change upon addition of PA $\beta \mathrm{N}$, whereas HDX was substantially increased upon CIP binding (Fig. 2a), possibly because the inhibitor weakens the coupling between the R1 and R2 TM domains. Notably, switch- loop spanning peptides with reduced HDX were detected when $\mathrm{PA} \beta \mathrm{N}$ was present, which may reflect a binding interaction and/ or structural stabilization (Fig. 2a, b and Supplementary Fig. 5).

Together, our HDX data support an inhibitory mode of action by which an EPI primarily acts to impart concerted restraint on AcrB structural dynamics, notably restricting the drug-binding pockets, connecting- and switch-loops.

Multi-copy 1- $\mu$ s long MD simulations of $A c r B$ WT bound to $\mathrm{PA} \beta \mathrm{N}$ confirmed that-in agreement with the previous literature ${ }^{6}$ -the inhibitor could stably bind to the DBP, straddling the switch-loop and establishing strong interactions with the hydrophobic trap (HT); a peculiar region of the DBP rich in phenylalanines and involved in EPI binding 4,36 (Supplementary Fig. 6).

To further understand the dynamics of AcrB in the presence of substrates, Root Mean Square Fluctuation (RMSF) and first hydration shell profiles were compared to the HDX-MS data. The average number of water molecules in the first amide $\mathrm{NH}$ solvation shell computed by MD simulations has been found to correlate well with $\mathrm{HDX}^{37}$. A reduced hydration shell should therefore imply reduced HDX, due to the decrease in specific interactions between amide $\mathrm{N}-\mathrm{H}$ bonds and the solvent. However, protein HDX is complex, with neighbouring residues having significant differences in their solvent interactions, this combined with the stark contrast between MD simulation and HDX-MS experimental time scales ( $\mu$ s to ms versus seconds to hours) means that a simple quantitative comparison can often be incomplete. Nevertheless, comparisons to MD calculated hydration profiles can provide informative qualitative interpretation of protein HDX.

$\mathrm{MD}$ analysis of $\mathrm{AcrB}^{\mathrm{WT}}-\mathrm{PA} \beta \mathrm{N}$ (T monomer) and apo $\mathrm{AcrB} \mathrm{WT}^{\mathrm{W}}$ ( $\mathrm{L}$ monomer) revealed that binding of $\mathrm{PA} \beta \mathrm{N}$ is accompanied by an overall rigidification of the protein (Supplementary Fig. 7; see also Supplementary Discussion and Supplementary Tables 2, 3), which involves large patches of the DBP, PBP, switch-loop, as well as the exit channel gate (EG), $\mathrm{CH} 1$, and $\mathrm{CH} 2$ channels $^{8}$. In particular: (i) regions containing residues belonging/adjacent to the switch-loop that were found to directly interact with $P A \beta N$ become more rigid in its presence, the extent of HDX protection upon PA $\beta \mathrm{N}$ binding (as revealed by the HDX-MS data) correlating with the formation of hydrogen bonds between the EPI and residues of (and nearby) the DBP; ii) the switch-loop itself (residues 615-620) features moderately enhanced hydration, whereas the nearby segments (residues 612-614 and 621-624) are overall dehydrated with respect to apo $A c r B \mathrm{WT}$. This supports an interaction between $\mathrm{PA} \beta \mathrm{N}$ and the switch-loop region, which we anticipate being a key factor in mediating the mode of action of this EPI. More generally, the structural stabilization that occurs upon PA $\beta \mathrm{N}$ binding might prevent local, as well as distal, functional movements that are key to substrate efflux along the transport pathway.

Overall, these data agree with a model for inhibitor action, which has been proposed to work by trapping AcrB in a conformation, possibly a T-like state, which prevents adequate functional rotation and substrate transport ${ }^{16}$.

EPI and antibiotic can dually bind to AcrB. To fully understand EPI action, it is essential to consider its activity in the presence of antibiotic substrates, especially considering the emerging importance of drug combination therapies for treating bacterial infection $^{38}$. Antibiotic susceptibility assays against $E$. coli confirmed the ability of the PAßN EPI to potentiate antibiotic activity (Fig. 2c). PA $\beta \mathrm{N}$ increased antibiotic susceptibility for a range of antimicrobial AcrB substrates (ciprofloxacin (CIP), tetracycline (TET), and chloramphenicol (CHL)) in a substrate-dependent 
a
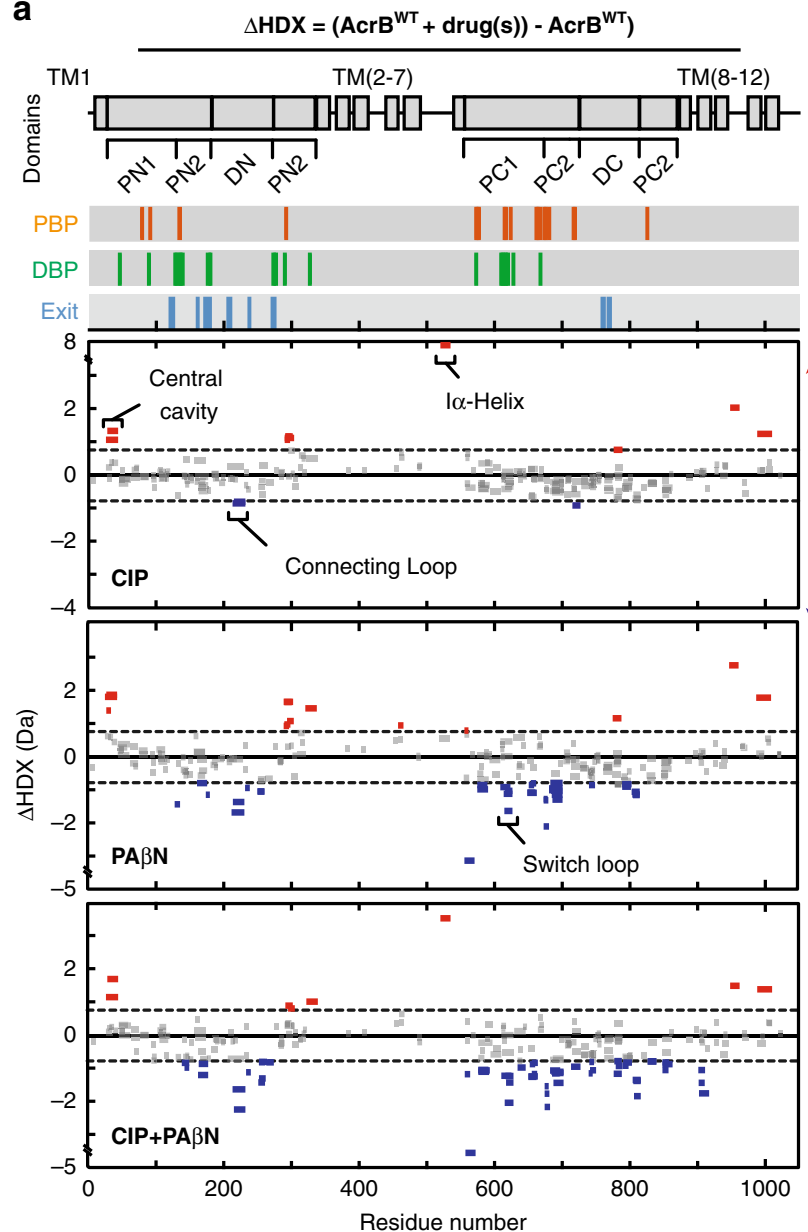

b

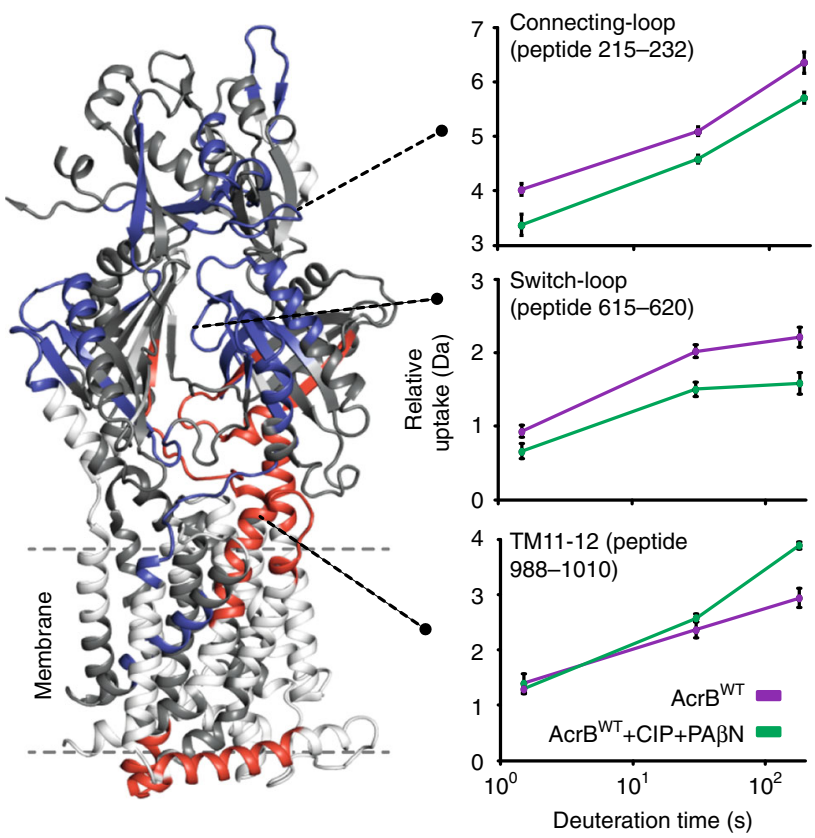

C

\begin{tabular}{|c|c|c|c|}
\hline \multirow[t]{2}{*}{$\begin{array}{l}\text { Escherichia coli } \\
\text { strain }\end{array}$} & \multicolumn{3}{|c|}{$\begin{array}{l}\text { Minimum Inhibitory Concentration } \\
\qquad(\mu \mathrm{g} / \mathrm{ml})\end{array}$} \\
\hline & CIP & TET & $\mathrm{CHL}$ \\
\hline ATCC 25922 & 0.008 & 1 & 4 \\
\hline 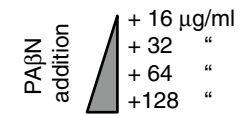 & $\begin{array}{c}0.004 \\
0.002 \\
0.001 \\
\leq 0.00012\end{array}$ & $\begin{array}{c}1 \\
0.5 \\
0.25 \\
\leq 0.008\end{array}$ & $\begin{array}{c}1 \\
1 \\
0.5 \\
0.25\end{array}$ \\
\hline
\end{tabular}

Fig. 2 Effect of EPI PA $\beta$ N on AcrB structural dynamics and function. a Sum differential HDX $(\Delta \mathrm{HDX})$ plots for different drug conditions ( $\Delta \mathrm{HDX}=$ $(A c r B W T+\operatorname{drug}(s))-A c r B W T)$ for all time points collected. Red signifies peptides with increased HDX between states and blue represents peptides with decreased HDX. 98\% confidence intervals are shown as grey dotted lines and grey data are peptides with insignificant $\triangle H D X$. All measurements were performed at least in triplicate. All supporting HDX-MS peptide data can be found in the Source Data file. $\mathbf{b} \Delta \mathrm{HDX}$ extent for (AcrBWT $+\mathrm{CIP}+\mathrm{PA} \beta \mathrm{N})-$ $A c r B W T$ is coloured onto the L-state monomer of AcrB (PDB:2HRT) using Deuteros ${ }^{34}$. Connecting-loop from the adjacent monomer is included. Uptake plot data are the average and standard deviation from repeated measurements $(n=3)$. c MIC assays of Escherichia coli in the presence of inhibitor and antibiotics. Values that demonstrate $>2$-fold reduction in MIC are in bold. Ciprofloxacin $=\mathrm{CIP}$, Tetracycline $=\mathrm{TET}$, Chloramphenicol $=\mathrm{CHL}$, and phenylalanine-arginine- $\beta$-naphthylamide $=\mathrm{PA} \beta \mathrm{N}$. MIC for PA $\beta \mathrm{N}$ alone is $256 \mu \mathrm{g} / \mathrm{ml}$.

manner, with better effectiveness observed at lower PAßN concentrations for CIP than for TET and CHL.

To explore the effect of an antibiotic on efflux inhibition we performed HDX-MS in the presence of both CIP and PA $\beta N$ $\left(\mathrm{AcrB} \mathrm{WT}^{\mathrm{WT}} \mathrm{CIP}-\mathrm{PA} \beta \mathrm{N}\right)$. We anticipated that the presence of equimolar CIP may interfere with $\mathrm{PA} \beta \mathrm{N}$ binding, thereby affecting its ability to prevent functionality of the transporter through dynamic restrain. This was not the case. The presence of CIP did not alter the action of the PA $\beta \mathrm{N}$ inhibitor, as revealed by the strikingly similar differential HDX profiles for both AcrBWT $\mathrm{PA} \beta \mathrm{N}$ and $\mathrm{AcrB}^{\mathrm{WT}}-\mathrm{CIP}-\mathrm{PA} \beta \mathrm{N}$ (Fig. 2a, b and Supplementary Fig. 5). Consequently, we investigated the possibility that PA $\mathrm{N}$ acts by outcompeting CIP binding to AcrB. To test this, we exploited the innate fluorescence of CIP to perform fluorescence polarization binding and competition assays ${ }^{39}$. Interestingly, we found that CIP binds with comparable affinity to both $\mathrm{AcrB}^{\mathrm{WT}}\left(K_{\mathrm{D}}\right.$ of $74.1 \pm 2.6 \mu \mathrm{M}$ from Su et al. $\left.{ }^{39}\right)$ and a preformed AcrB ${ }^{\text {WT }}-\mathrm{PA} \beta \mathrm{N}$ complex $\left(K_{\mathrm{D}}\right.$ of $\left.67.3 \pm 13.2 \mu \mathrm{M}\right)$ (Fig. $\left.3 \mathrm{a}\right)$, and that titration of PA $\beta \mathrm{N}$ EPI could not effectively outcompete $\mathrm{CIP}$ binding from a $\mathrm{AcrB}^{\mathrm{WT}}$-CIP complex (Fig. 3b). These data suggest that antibiotic and inhibitor may be able to simultaneously bind at different (sub)sites within the voluminous DBP.

To further support this hypothesis, we performed blind docking calculations and $\mathrm{MD}$ simulations on $\mathrm{AcrB}{ }^{\mathrm{WT}}-\mathrm{PA} \beta \mathrm{N}-\mathrm{CIP}$ (Supplementary Figs. 8, 9). Importantly, both drugs stably bind to the DBP within the T-state monomer, with PA $\beta \mathrm{N}$ partly occupying the HT and CIP lying in proximity of the PBP/DBP interface (Fig. 3c). The simultaneous binding of CIP and PA $\beta \mathrm{N}$ has similar effects on the flexibility and hydration of the DBP as the binding of PA $\beta \mathrm{N}$ only (Fig. 2a, b and Supplementary Fig. 10; see Supplementary Discussion). Several interactions contribute to stabilize this configuration, including the formation of hydrogen bonds between the substrates and several residues of the DBP (Supplementary Table 4) and stable intermolecular hydrogen bonds between the two ligands (Supplementary Fig. 9).

Overall, our data support the hypothesis that PA $\beta \mathrm{N}$ does not compete or prevent antibiotic binding (competitive inhibition). Instead, we propose that it inhibits AcrB function by enforcing a more restrained state, thus, reducing the frequency and 
a
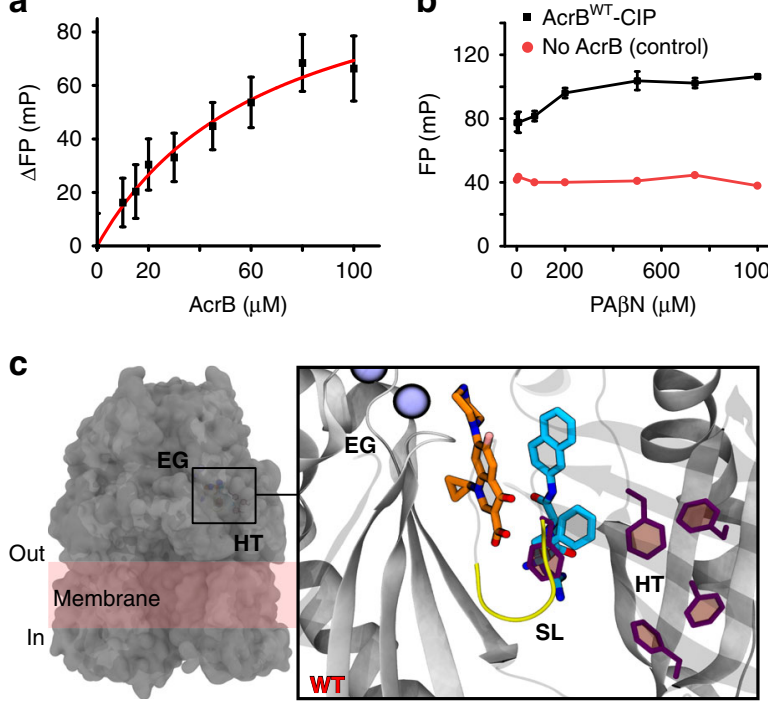

Fig. 3 Dual binding of ciprofloxacin antibiotic and PA $\beta N$ inhibitor to the DBP of AcrBWT. a Binding of CIP by AcrBWT in the presence of $150 \mu \mathrm{M}$ of $\mathrm{PA} \beta \mathrm{N}$ as determined by a fluorescence polarization assay performed by Su et al. ${ }^{39}$. CIP was maintained at $1.5 \mu \mathrm{M}$ throughout and its emission wavelength measured at $415 \mathrm{~nm}$. Reported data are the average and standard deviation from independent measurements $(n=3)$ and were fitted to a hyperbola function (FP $=\left(B_{\max }{ }^{\star}[\right.$ protein $\left.]\right) /\left(K_{D}+[\right.$ protein $\left.]\right)$, $\left.R^{2}=0.98\right)$. $\mathbf{b}$ Binding competition assay between $\mathrm{PA} \beta \mathrm{N}$ and $\mathrm{CIP}$ for $A c r B W T$. PA $\beta N$ was non-fluorescent in the experimental conditions. Data are the average and standard deviation from independent measurements $(n=3)$. c Molecular docking and multi-copy $\mu$ s-long MD simulations reveal stable interactions of CIP (orange) and PA $\beta N$ (cyan) to AcrBWT T-state monomer and show their likely binding locations. $E G=$ exit channel gate (blue spheres), $\mathrm{SL}=$ switch-loop (yellow), and $\mathrm{HT}=$ hydrophobic trap (purple). All computational data can be found in Supplementary Table 2 and Supplementary Figs. 8, 9

magnitude of the conformational changes within the substrate translocation path. Its effectiveness being substrate dependent.

MDR-conferring G288D mutation affects drug-binding pocket dynamics. We next turned our attention to the substitution mutation, G288D ( $\mathrm{AcrB}^{\mathrm{G} 288 \mathrm{D}}$ ), which was found to cause resistance to some drugs (e.g. CIP) in Salmonella, but susceptibility to others (e.g. minocycline $(\mathrm{MIN}))^{30}$. G288 is a highly conserved residue in Enterobacteriaceae ${ }^{30}$, suggesting an important structural role, and is present aside the HT within the DBP (Fig. 4a). We found that the G288D substitution does not alter the oligomeric state, average secondary structure content or thermal stability of AcrB, as judged by native mass spectrometry and circular dichroism (Supplementary Fig. 2). Yet, our HDX analysis revealed that the G288D mutation has a noticeable effect on the structural dynamics of AcrB. We observed that, when no drugs were present, the G288D mutation caused increased HDX for several peptides spanning the PN2 region of the protein, but decreased HDX within the PC1/PC2 regions and the connectingloop (Fig. 4b). Seemingly, this single-point mutation can cause a long-range change to the structural dynamics of AcrB, possibly reflecting global conformational changes in its substrate-free state.

Upon substrate binding, $\mathrm{PA} \beta \mathrm{N}$ caused reduced HDX within the PC1/PC2 regions for AcrB ${ }^{\mathrm{G} 288 \mathrm{D}}$ (Supplementary Fig. 11), as was observed for $A c r B W T$ (Fig. 2a), with $A c r B{ }^{G 288 D}-P A \beta N$ having increased HDX reduction within PC1 and R2 (TM 7-12) domains in comparison to $\mathrm{AcrB}^{\mathrm{WT}}-\mathrm{PA} \beta \mathrm{N}$ (Fig. 4b). This supports that the PA $\beta \mathrm{N}$ EPI affects the dynamics of the different AcrB genotypes in a similar manner, with $\mathrm{AcrB}^{\mathrm{G} 288 \mathrm{D}}-\mathrm{PA} \beta \mathrm{N}$ possibly undergoing further restraint than $\mathrm{AcrB}^{\mathrm{WT}}-\mathrm{PA} \beta \mathrm{N}$. Whereas CIP caused increased HDX throughout extensive regions of $A c r B G 288 \mathrm{D}$ (Supplementary Fig. 11), bringing the $\mathrm{PC} 1 / \mathrm{PC} 2$ and DC regions of $\mathrm{AcrB} \mathrm{G}^{\mathrm{2} 88 \mathrm{D}}$ closer in parity with $\mathrm{AcrB}^{\mathrm{WT}}$ for both CIP and CIP-PAßN conditions (Fig. $4 \mathrm{~b}$ ).

Markedly, in the apo form and for all three substrate conditions tested (CIP, PA $\beta \mathrm{N}$, and CIP-PA $\beta \mathrm{N}$ ), the G288D substitution consistently caused increased HDX within the PN2 region and decreased HDX of the connecting-loop (Fig. 4b). Signifying that these effects are retained even upon substrate binding and, due to their close structural proximity, may relate to concerted changes to the dynamics of the substrate translocation pathway (Fig. 4c).

PA $\beta$ N EPI inhibits both wildtype and G288D AcrB. MD simulations of $A c r B$ G288D in the presence of $P A \beta N$ were performed to better understand how the G288D mutation affects EPI interactions with the drug-binding pockets. PA $\beta \mathrm{N}$ was found to bind to the HT of $A c r B{ }^{G 288 D}$, interacting with the mutated D288 residue through the formation of direct and water-mediated hydrogen bonds (Supplementary Fig. 12 and Supplementary Table 5). Interactions with the aromatic residues of the HT involve hydrophobic stacking as well as cation- $\pi$ attraction, not observed in $\mathrm{AcrB}^{\mathrm{WT}}-\mathrm{PA} \beta \mathrm{N}$, and possibly promoted by the direct interaction of the inhibitor with residue D288 (Supplementary Table 6; see Supplementary Discussion). This interaction is supported by the reduced HDX found within D288 containing peptides of AcrB ${ }^{\mathrm{G} 288 \mathrm{D}}-\mathrm{PA} \beta \mathrm{N}$ (Supplementary Fig. 11b). Moreover, in accordance with HDX-MS, the switch-loop and the surrounding DBP region undergo further dehydration in

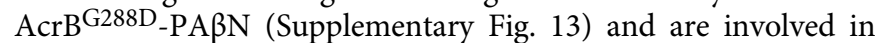
high-occurrence interactions with $\mathrm{PA} \beta \mathrm{N}$, similar to those formed within $\mathrm{AcrB}^{\mathrm{WT}}-\mathrm{PA} \beta \mathrm{N}$ (Supplementary Tables 3,6 ). These data, together with the direct interactions detected between the EPI and regions of the switch-loop, support the hypothesis that stabilization of the latter has a role for inhibitor mode of action of $\mathrm{PA} \beta \mathrm{N}$ both in $\mathrm{AcrB}^{\mathrm{WT}}$ and in $\mathrm{AcrB} \mathrm{G}^{\mathrm{G} 28 \mathrm{D}}$.

Similar conclusions emerged from the comparison between

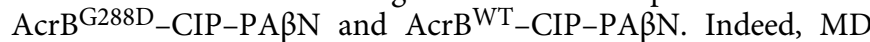
simulations of the former complex revealed that, even upon G288D substitution, CIP and PA $\beta \mathrm{N}$ can stably occupy the DBP at the same time (Fig. 5a). As in AcrB ${ }^{W T}$-CIP-PAßN, stabilizing interactions include several substrate contacts with the $A c r B$ G288D protein, also involving D288 (Supplementary Figs. 14, 15 and Supplementary Table 7), as well as intermolecular hydrogen bonds between the two ligands (Supplementary Table 8).

Fluorescence polarization binding and competition assays support that a ternary $\mathrm{AcrB}^{\mathrm{G} 288 \mathrm{D}}-\mathrm{CIP}-\mathrm{PA} \beta \mathrm{N}$ is also possible (Fig. 5b, c): (i) CIP binds to a preformed $\mathrm{AcrB}^{\mathrm{G} 288 \mathrm{D}}-\mathrm{PA} \beta \mathrm{N}$ complex $\left(K_{\mathrm{D}}\right.$ of $\left.22.7 \pm 2.9 \mu \mathrm{M}\right)$ with similar, albeit slightly higher, affinity compared to CIP binding to AcrB ${ }^{\mathrm{WT}}-\mathrm{PA} \beta \mathrm{N}$ ( $K_{\mathrm{D}}$ of $67.3 \pm 13.2 \mu \mathrm{M}$ ); (ii) titration of the PA $\beta \mathrm{N}$ inhibitor could not effectively outcompete CIP binding from $\mathrm{AcrB}^{\mathrm{G} 288 \mathrm{D}}$-CIP, as was found for AcrB ${ }^{W T}-C I P$ (Fig. 3b). Taken together, the fluorescence polarization binding assays and $\mathrm{MD}$ simulation data advocate that $A c r B$ G288D is inhibited by $P A \beta N$ in a similar manner as $\mathrm{AcrB}$ WT .

These findings were supported by bacterial susceptibility assays on E. coli containing overexpressed $A c r B^{G 288 D}$. $A_{c r B}{ }^{G 288 D}$ was previously discovered within Salmonella clinical isolates ${ }^{30}$ and found to have increased and decreased susceptibility to MIN and CIP antibiotics, respectively. We chose, therefore, to study these AcrB substrates in the presence of the inhibitor PA $\beta \mathrm{N}$ to observe 
a

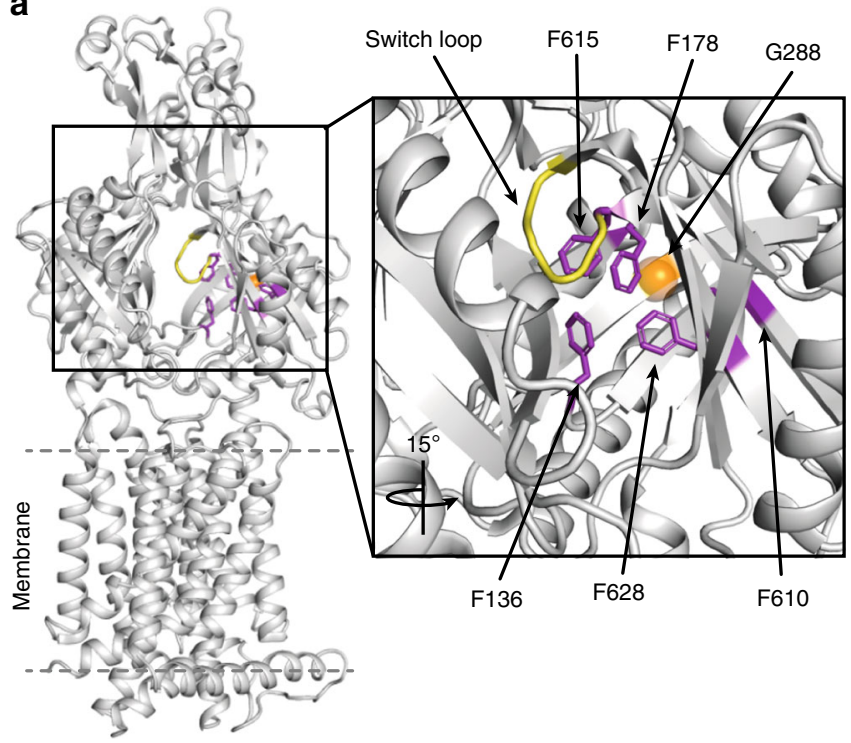

C

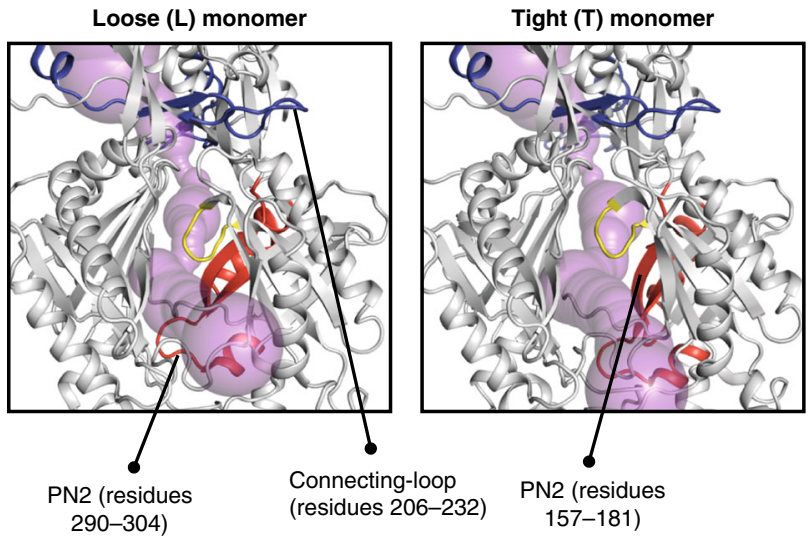

b
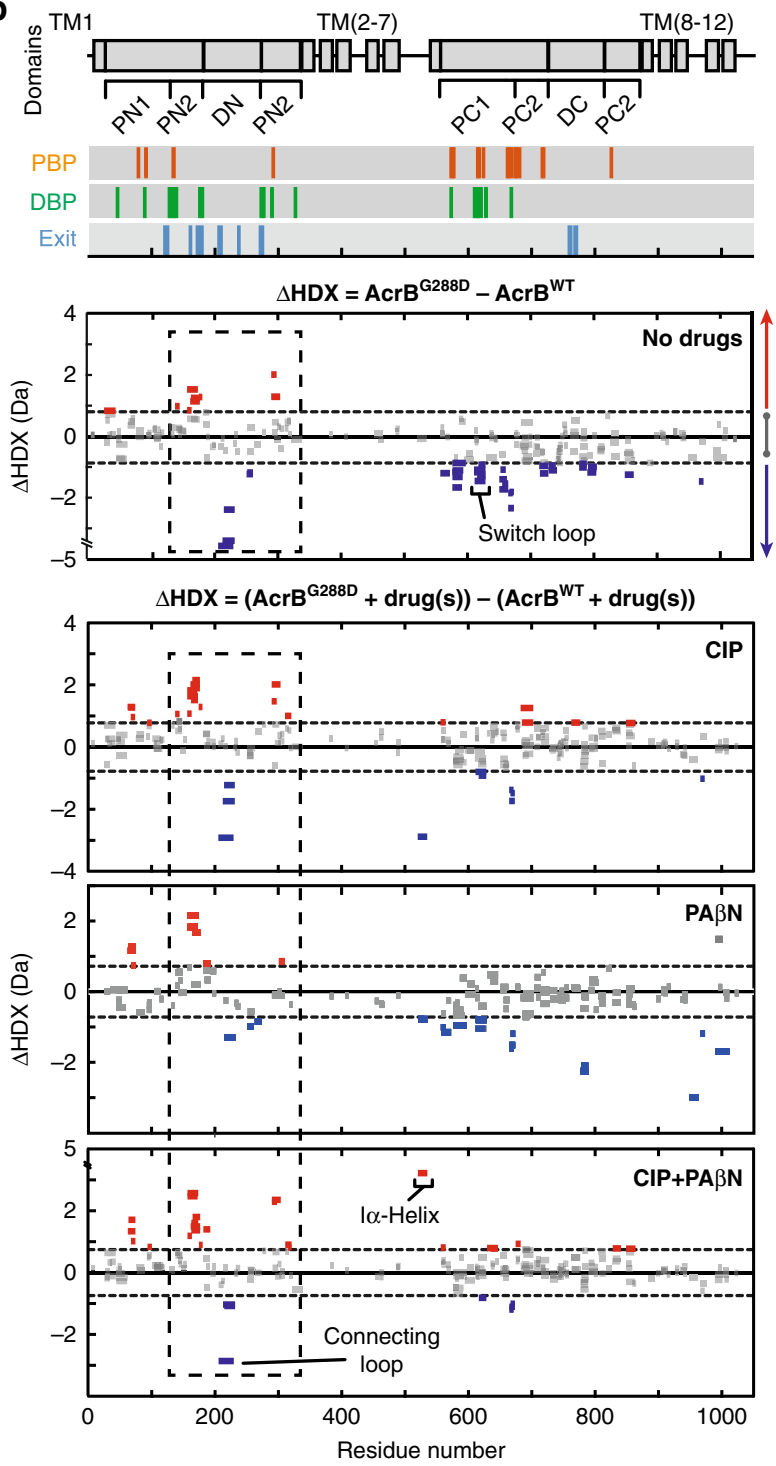

Fig. 4 Influence of MDR mutation G288D on AcrB structural dynamics. a In this zoom-in view of the DBP, within the L-state monomer of AcrB (PDB:2HRT), the G288 position is shown as an orange sphere amongst the hydrophobic trap (purple) and alongside the switch loop (yellow). $\mathbf{b}$ Differential $\mathrm{HDX}(\triangle \mathrm{HDX})$ plots for $\mathrm{AcrB} \mathrm{B}^{\mathrm{G} 88 \mathrm{D}}$ and $\mathrm{AcrB}{ }^{W T}$ with and without drugs. All data reported as in Fig. $2.98 \%$ confidence intervals are shown as grey dotted lines and grey data are peptides with insignificant $\triangle \mathrm{HDX}$. All measurements were performed at least in triplicate. All supporting HDX-MS peptide data can be found in the Source Data file. (c) Shared regions surrounding the DBP whose HDX are increased (red) and decreased (blue) in a substrate-independent manner (highlighted by the dashed boxes in Fig. $4 \mathrm{~b}$ ) are shown on both L- and T-state monomers of AcrB (PDB:2HRT). The connecting-loop from the adjacent monomer is included. Substrate efflux pathways (magenta) for the periplasmic entrance are depicted using CAVER ${ }^{68}$.

what, if any, effect would be seen with the different AcrB genotypes. PA $\beta \mathrm{N}$ incubation led to increased MIN and CIP antibiotic susceptibility for both $A c r B{ }^{W T}$ and AcrB ${ }^{\mathrm{G} 288 \mathrm{D}}$ (Fig. 5d). $A c r B^{G 288 D}$ being more susceptible to $P A \beta N$ than $A c r B$ WT. The decreased susceptibility of $\mathrm{AcrB}^{\mathrm{G} 288 \mathrm{D}}$ to CIP, found in Salmonella ${ }^{30}$, was not recapitulated in our assays using the laboratory $E$. coli strain MG1655. This may be due to CIP efflux via another transporter found in E. coli but not in Salmonella. However, the associated increased susceptibility to MIN was observed (Fig. 5d), supporting that G288D has a profound impact on AcrB substrate efflux within both E. coli and Salmonella.

\section{Discussion}

In summary, we found that binding of an EPI, PA $\beta N$, restricts AcrB dynamics and could not be outcompeted by an antibiotic, $\mathrm{CIP}$, whose activity it potentiates. Fluorescence binding, MD simulations, and docking studies supporting the existence of a ternary protein-EPI-antibiotic complex. Endorsing the theory that RND-pump inhibitors act through an "altered-dynamics" mechanism, obstructing the translocation of substrates rather than preventing their binding and recognition.

Furthermore, we reveal that an MDR-conferring AcrB drugpocket substitution, G288D ${ }^{30}$, modifies the structural dynamics of the translocation pathway in a substrate-independent manner. Our previous MD simulations ${ }^{30}$ show that the G288D substitution increases the gyration radius and hydration at and around the DBP. This disruption could subsequently lead to the observed allosteric action on farther AcrB regions. In turn, these changes may alter the energetic barrier for substrate binding and transport during functional rotation and, consequently, be the ultimate cause for the altered substrate efflux caused by this mutation. 
a

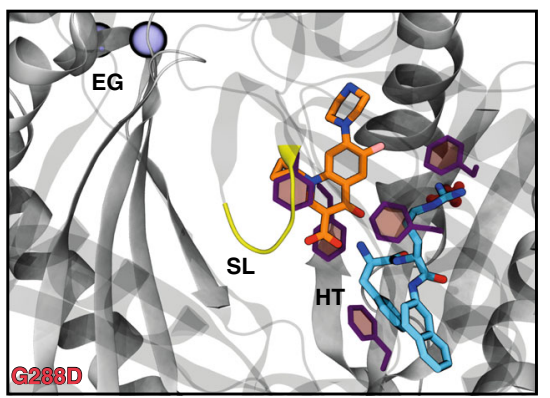

b
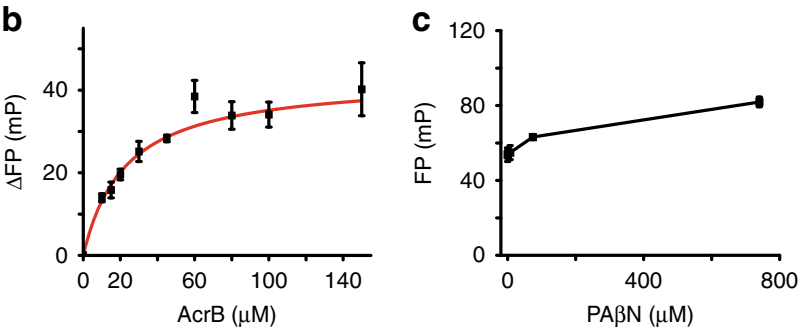

d

\begin{tabular}{|c|c|c|c|c|c|}
\hline \multirow{2}{*}{$\begin{array}{c}\text { Escherichia coli } \\
\text { strain }\end{array}$} & \multicolumn{5}{|c|}{ Minimum Inhibitory Concentration $(\mu \mathrm{g} / \mathrm{ml})$} \\
\hline & CIP & $\begin{aligned} & \text { CIP } \\
+ & \text { PA } \beta N^{\dagger}\end{aligned}$ & MIN & $\begin{aligned} & \mathrm{MIN} \\
+ & \mathrm{PA} \mathrm{N}^{\dagger}\end{aligned}$ & $\mathrm{PA} \beta \mathrm{N}$ \\
\hline MG1655 & 0.015 & 0.008 & 2 & 0.25 & 512 \\
\hline MG1655 $\triangle$ acrB & 0.004 & $<0.002$ & 0.5 & 0.06 & 128 \\
\hline$"+p B R-A c r B{ }^{W T}$ & 0.06 & 0.015 & $>2$ & 0.25 & 512 \\
\hline$"+p B R-A c r B^{G 288 D}$ & 0.03 & 0.004 & 1 & 0.06 & 256 \\
\hline
\end{tabular}

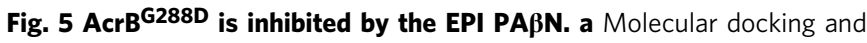
multi-copy $\mu$ s-long MD simulations reveal stable interactions of CIP (orange) and PA $\beta N$ (cyan) to $A c r B G 288 D$ T-state monomer and show their likely binding locations. The pose and its orientation are the same as shown for AcrBWT in Fig. 3c. EG = exit channel gate (blue spheres), $\mathrm{SL}=$ switchloop (yellow), and $\mathrm{HT}=$ hydrophobic trap (purple). All computational data, including binding free energies can be found in Supplementary Table 5 and Supplementary Figs. 8, 14-16. b Binding of CIP by AcrB ${ }^{\mathrm{G} 288 \mathrm{D}}$ in the presence of $150 \mu \mathrm{M}$ of $\mathrm{PA} \beta \mathrm{N}$ as determined by a fluorescence polarization assay performed by Su et al. ${ }^{39}$. All data are fit as in Fig. 3a $\left(R^{2}=0.99\right)$. Data are the average and standard deviation from independent measurements $(n=3)$. c Binding competition assay between PA $\beta N$ and $C I P$ for $A c r B$ WT . Data are the average and standard deviation from independent measurements $(n=3)$. $\mathbf{d}$ MIC assays of Escherichia coli containing AcrBWT or $A c r B{ }^{G 288 D}$ in the presence of PA $\beta N$ and antibiotics. AcrB was overexpressed in MG1655 $\triangle a c r B$ from a pBR322 plasmid containing its corresponding $a c r A B$ genes, natural promoter and "marbox" sequence. Minocycline $=$ MIN, ciprofloxacin $=C I P$, and phenylalanine-arginine- $\beta$ naphthylamide $=P A \beta N$. ${ }^{\dagger} P A \beta N$ was added at a concentration of $50 \mu \mathrm{g} / \mathrm{ml}$.

Here, to examine the structural dynamics of AcrB we performed HDX-MS within DDM detergent micelles and supported our findings with $\mathrm{MD}$ simulations completed within POPE/ POPG (2:1 ratio) lipid bilayers. Detergent and amphipol membrane mimetics have been used extensively to obtain structural information for the determination of drug binding interactions and efflux mechanisms of AcrB. However, these systems do not provide a lipid environment, which can modulate membrane protein structure and function ${ }^{40}$. Recent studies have used SMA lipid particle (SMALP) technology and liposome reconstitution to capture AcrB within a lipid environment and solved its structure at high-resolution using cryo-EM ${ }^{41-43}$. The resulting structures were largely consistent with high-resolution crystal structures solved in DDM detergent micelles, with homotrimeric AcrB conformation remaining the same even when the surrounding membranes display different curvatures ${ }^{43}$. This coupled with the agreement found between our HDX-MS data and MD simulations support that the structural dynamic behaviour uncovered here informs on the native protein state, although further studies investigating the effect of lipids on its dynamics are necessary to understand the system in its entirety.

We anticipate that the findings reported here will be important not only for establishing the general role of structural dynamics in modulating AcrB multidrug binding and efflux, which are hard to elucidate from biochemical and high-resolution structural data alone, but also for defining how naturally occurring mutations and EPI interactions affect its structure function.

\section{Methods}

Plasmid construction. An overexpression plasmid containing AcrB with a Cterminal 6xHistidine tag (AcrB-6xHis) was constructed from a pET15b-AcrBsGFP-6xHis plasmid from Reading et al. ${ }^{44}$. Briefly, the sGFP sequence was deleted and a 6 xHis-tag placed at the C-terminus of AcrB followed by a stop codon using the Q $5^{\oplus}$ site-directed mutagenesis kit (New England Biolabs)-AcrB contains two Histidine residues at its C-terminus, therefore, this construct resulted in AcrB having an $8 \mathrm{xHistidine}$ tag. The G288D mutation was then generated from this pET15b-AcrB-6xHis plasmid using the $\mathrm{Q}^{\circledR}{ }^{\circledR}$ site-directed mutagenesis kit (New England Biolabs). All constructs were verified by DNA sequencing (Eurofins MWG). Primers used are reported in Supplementary Table 9.

pBR322-AcrB plasmids were generated for bacterial susceptibility assays. Briefly, pBR322 was linearized with HindIII and EcoRI restriction enzymes (New England Biolabs). $a c r A B$ genes with its natural promoter, including the "marbox" sequence, was then amplified from K-12 Escherichia coli chromosomal DNA (Zyagen Labs) and cloned into the pBR322 vector using In-Fusion ${ }^{\circledast} \mathrm{HD}$ cloning (Takara Bio). A 6xHistidine tag sequence was included in the reverse primer to provide a $6 \mathrm{xHis}$ tag at the C-terminus of $\mathrm{AcrB}\left(\mathrm{pBR}-\mathrm{AcrB}{ }^{\mathrm{WT}}\right)$. The G288D mutation was generated from this pBR-AcrB ${ }^{W T}$ plasmid using the $\mathrm{Q} 5^{\oplus}$ sitedirected mutagenesis kit (New England Biolabs). All constructs were verified by primer walking DNA sequencing (Eurofins MWG). Primers used are reported in Supplementary Table 9.

AcrB overexpression and purification. pET15b-AcrB-6xHis plasmid containing AcrB wildtype $(A c r B$ WT $)$ or G288D mutant $\left(A c r B{ }^{G 288 D}\right)$ was transformed into $C 43$ (DE3) $\triangle a c r B:: K a n R$ E. coli cells. $7 \mathrm{ml}$ of an overnight LB culture was added to $1 \mathrm{~L}$ of pre-warmed LB culture containing $100 \mu \mathrm{g} / \mathrm{ml}$ ampicillin and $30 \mu \mathrm{g} / \mathrm{ml}$ kanamycin and grown at $37^{\circ} \mathrm{C}$ until an OD of $0.6-0.8$ was reached. The culture was induced with $1 \mathrm{mM}$ IPTG and grown for $16-18 \mathrm{~h}$ at $18^{\circ} \mathrm{C}$. At which point the cells were harvested by centrifugation at $4200 \times g$ for $30 \mathrm{~min}$ and washed with ice-cold phosphate buffer saline (PBS).

Cell pellets were immediately resuspended in buffer A $(50 \mathrm{mM}$ sodium phosphate, pH 7.4, $300 \mathrm{mM}$ sodium chloride) and supplemented with a protease inhibitor tablet (Roche), $100 \mu \mathrm{M}$ PMSF, $1 \mu \mathrm{l}$ Benzonase, and $5 \mathrm{mM}$ betamercaptoethanol ( $\beta$-ME). The cell suspension was then passed twice through a microfluidizer processor (Microfluidics) at 25,000 psi and $4{ }^{\circ} \mathrm{C}$. Insoluble material was removed by centrifugation at $20,000 \times g$ for $30 \mathrm{~min}$ at $4^{\circ} \mathrm{C}$. Membranes were then pelleted from the supernatant by centrifugation at $200,000 \times g$ for $1 \mathrm{~h}$ at $4^{\circ} \mathrm{C}$. Membrane pellets were resuspended to $40 \mathrm{mg} \mathrm{ml}^{-1}$ in ice-cold buffer A supplemented with a protease inhibitor tablet (Roche) and $100 \mu \mathrm{M}$ PMSF, and homogenized using a Potter-Elvehjem Teflon pestle and glass tube.

AcrB was extracted from homogenized membranes by overnight incubation with $1 \%(\mathrm{w} / \mathrm{v}) n$-dodecyl- $\beta$-D-maltoside (DDM) detergent (Anatrace) at $4{ }^{\circ} \mathrm{C}$ with gentle agitation. Insoluble material was then removed by centrifugation at 100,000 $\mathrm{x} g$ for $1 \mathrm{~h}$ at $4{ }^{\circ} \mathrm{C}$. The sample was then filtered through a $0.22 \mu \mathrm{m}$ filter (Fisher Scientific) and loaded onto a $1 \mathrm{ml}$ HiTrap column (GE Healthcare) equilibrated in buffer B (50 mM sodium phosphate, pH 7.4, $300 \mathrm{mM}$ sodium chloride, $20 \mathrm{mM}$ imidazole, $10 \%(\mathrm{w} / \mathrm{v})$ glycerol, $0.03 \%(\mathrm{w} / \mathrm{v}) \mathrm{DDM})$. The column was washed with 5 CVs of buffer B and then with $10 \mathrm{CVs}$ of buffer $\mathrm{C}(50 \mathrm{mM}$ sodium phosphate, $\mathrm{pH}$ 7.4, $300 \mathrm{mM}$ sodium chloride, $20 \mathrm{mM}$ imidazole, $10 \%(\mathrm{w} / \mathrm{v})$ glycerol, $1 \%(\mathrm{w} / \mathrm{v})$ octyl glucose neopentyl glycol (OGNG) (Generon)) - an OGNG detergent wash facilitates the removal of bound lipopolysaccharide (LPS), as determined previously 45 (Supplementary Fig. 2c-e). The column was then washed with $20 \mathrm{CVs}$ of Buffer B containing $50 \mathrm{mM}$ imidazole. AcrB was then eluted with Buffer B containing $500 \mathrm{mM}$ imidazole and directly injected onto a Superdex 75 10/600 GL size exclusion chromatography (SEC) column (GE Healthcare) equilibrated in buffer D (50 mM sodium phosphate, $\mathrm{pH} 7.4,150 \mathrm{mM}$ sodium chloride, $10 \%(\mathrm{w} / \mathrm{v})$ glycerol, $0.03 \%(\mathrm{w} / \mathrm{v}) \mathrm{DDM})$. A flow rate of $1 \mathrm{ml} / \mathrm{min}$ was used during HiTrap and SEC purification. Peak fractions eluted from the SEC column containing pure AcrB were pooled, spin concentrated using a $100 \mathrm{~K} \mathrm{MWCO} \mathrm{concentrator} \mathrm{(Amicon}{ }^{\oplus}$ ), and spin filtered before being flash frozen and stored at $-80^{\circ} \mathrm{C}$. SDS-PAGE electrophoresis was used to assess AcrB purification and protein concentration was 
calculated using a Cary 300 Bio UV-Vis spectrophotometer (Varian) with a calculated extinction coefficient ${ }^{46}$ of $\varepsilon_{280}=89,730 \mathrm{M}^{-1} \mathrm{~cm}^{-1}$.

Circular dichroism spectroscopy. Circular dichroism spectroscopy (CD) spectra were recorded with an Aviv Circular Dichroism spectrophotometer, Model 410 (Biomedical Inc., Lakewood, NJ, USA), with specially adapted sample detection to eliminate scattering artefacts. Multiple CD scans were averaged, the buffer background subtracted, and zeroed and minimally smoothed using $\mathrm{CDTool}^{47}$. A final protein concentration of $0.5-1.5 \mathrm{mg} \mathrm{ml}^{-1}$ was used in a quartz rectangular or circular Suprasil demountable cell (Hellma Analytics). For thermal protein unfolding the mean residue ellipticity at $222 \mathrm{~nm}$ was monitored with increasing temperature.

Native mass spectrometry. Purified AcrB was buffer exchanged into MS buffer (200 mM ammonium acetate, $\mathrm{pH} 7.4,0.03 \%(\mathrm{w} / \mathrm{v})$ DDM or Triton X-100) using a centrifugal buffer exchange device (Micro Bio-Spin 6, Bio-Rad) according to the manufacturer's instructions ${ }^{48}$. Native mass spectrometry experiments were performed either on a Synapt G2-Si mass spectrometer (Waters) or a Thermo Scientific Q Exactive UHMR hybrid Quadrupole-orbitrap mass spectrometer.

For experiments on the Synapt G2-Si mass spectrometer the instrument settings used were: $1.5 \mathrm{kV}$ capillary voltage, source temperature of $25^{\circ} \mathrm{C}$, argon trap collision gas, $180 \mathrm{~V}$ trap collision voltage, $120 \mathrm{~V}$ cone voltage, and $50 \mathrm{~V}$ source offset. Data were processed and analyzed using MassLynx v.4.1 (Waters).

Native mass spectrometry data on a Thermo Scientific Q Exactive UHMR hybrid Quadrupole-orbitrap mass spectrometer was acquired at resolving power 8750 at $\mathrm{m} / \mathrm{z} 400$ in the $\mathrm{m} / \mathrm{z}$ range $2000-30.000$. The instrument was optimized for transmission and desolvation of integral membrane proteins. Critical parameters throughout were relative pressure of 6 , capillary temperature $250^{\circ} \mathrm{C}$, S-Lens RF level 0 , in-source trapping 200 and the HCD energy was $300 \%$. Data were analyzed by the use of Xcalibur software 4.3 and Biopharma Finder 3.1 (both Thermo Fisher Scientific). Deconvoluted spectra were acquired using Biopharma Finder 3.1 in sliding window mode using the following settings: Output mass range $10,000-100,000 \mathrm{Da}$, deconvolution mass tolerance $10 \mathrm{ppm}$, sliding window merge tolerance $30 \mathrm{ppm}$ and minimal number of detected intervals.

\section{Preparation of ligands for hydrogen/deuterium mass spectrometry. Cipro-} floxacin (CIP) antibiotic and Phe-Arg- $\beta$-naphthylamide dihydrochloride (PA $\beta N$ ) inhibitor were both purchased from Sigma Aldrich. Stock concentrations of CIP $(10 \mathrm{mg} / \mathrm{ml})$ and $P A \beta \mathrm{N}(10 \mathrm{mg} / \mathrm{ml})$ were prepared in $0.1 \mathrm{~N} \mathrm{HCl}$ and water, respectively. As demonstrated previously ${ }^{49}$, a primary consideration before carrying out HDX-MS is to ensure close to full binding to the target protein under deuterium exchange conditions; particularly for low-affinity ligands (dissociation constants, $k_{\mathrm{D}}$, in the $\mu \mathrm{M}$ range or lower). CIP and PA $\beta \mathrm{N}$ both possess moderate affinity binding to AcrB within DDM detergent micelles (CIP with a $k_{\mathrm{D}}$ of $74.1 \pm$ $2.6 \mu \mathrm{M}$, as measured by fluorescence polarization ${ }^{39}$ and PA $\beta \mathrm{N}$ with a $k_{\mathrm{D}}$ of $15.72 \pm$ $3.0 \mu \mathrm{M}$, as measured by surface plasmon resonance ${ }^{50}$ ). To achieve sufficient ligand binding saturation under deuterium exchange conditions AcrB was first incubated in the presence of saturating concentrations of ligand for 30 minutes on ice, before dilution into deuterated buffer containing saturating concentrations of ligand (740 $\mu \mathrm{M}, \sim 1000: 1$ ligand to AcrB ratio) for deuterium exchange experiments.

Hydrogen/deuterium mass spectrometry. Hydrogen/deuterium mass spectrometry (HDX-MS) was performed on an HDX nanoAcquity ultra-performance liquid chromatography (UPLC) Synapt G2-Si mass spectrometer system (Waters Corporation). Optimized peptide identification and peptide coverage for AcrB was performed from undeuterated controls. The optimal sample workflow for HDXMS of AcrB was as follows: $5 \mu \mathrm{l}$ of AcrB $(15 \mu \mathrm{M})$ was diluted into $95 \mu \mathrm{l}$ of either buffer $\mathrm{D}$ or deuterated buffer $\mathrm{D}$ at $20^{\circ} \mathrm{C}$. After fixed times of deuterium incubation samples were mixed with $100 \mu \mathrm{l}$ of formic acid-DDM quench solution to provide a quenched sample at pH 2.5 and final $0.075 \%$ (w/v) DDM concentration. $80 \mu \mathrm{l}$ of the quenched sample was then loaded onto a $50 \mu \mathrm{l}$ sample loop before being injected onto an online Enzymate ${ }^{\mathrm{xx}}$ pepsin digestion column (Waters) in $0.1 \%$ formic acid in water (at a flow rate of $200 \mu \mathrm{l} / \mathrm{min}$ ) maintained at $20^{\circ} \mathrm{C}$. The peptic fragments were trapped onto an Acquity BEH C18 $1.7 \mu \mathrm{M}$ VANGUARD precolumn (Waters) for $3 \mathrm{~min}$. The peptic fragments were then eluted using an $8-40 \%$ gradient of $0.1 \%$ formic acid in acetonitrile at $40 \mu \mathrm{l} / \mathrm{min}$ into a chilled Acquity UPLC BEH C18 $1.7 \mu \mathrm{M} 1.0 \times 100 \mathrm{~mm}$ column (Waters). The trap and UPLC columns were both maintained at $0^{\circ} \mathrm{C}$. The eluted peptides were ionized by electrospray into the Synapt G2-Si mass spectrometer. $\mathrm{MS}^{\mathrm{E}}$ data was acquired with a 20-30 V trap collision energy ramp for high-energy acquisition of product ions. Argon was used as the trap collision gas at a flow rate of $2 \mathrm{~mL} / \mathrm{min}$. Leucine enkephalin was used for lock mass accuracy correction and the mass spectrometer was calibrated with sodium iodide. The online Enzymate ${ }^{\mathrm{mm}}$ pepsin column was washed three times with pepsin wash $(1.5 \mathrm{M} \mathrm{Gu}-\mathrm{HCl}, 4 \% \mathrm{MeOH}, 0.8 \%$ formic acid), as recommended by the manufacturer, and a blank run was performed between each sample to prevent significant peptide carry-over between runs.

All deuterium time points and controls were performed in triplicate. Sequence identification was performed from $\mathrm{MS}^{\mathrm{E}}$ data of digested undeuterated samples of AcrB using the ProteinLynx Global Server 2.5.1 software. The output peptides were then filtered using DynamX (v. 3.0) using the following filtering parameters: minimum intensity of 1000, minimum and maximum peptide sequence length of 4 and 25 , respectively, minimum MS/MS products of 1 , minimum products per amino acid of 0.12 , and a maximum $\mathrm{MH}^{+}$error threshold of $15 \mathrm{ppm}$. Additionally, all the spectra were visually examined and only those with a suitable signal to noise ratios were used for analysis. The amount of relative deuterium uptake for each peptide was determined using DynamX (v. 3.0) and was not corrected for back exchange ${ }^{32}$. The relative fractional uptake (RFU) was calculated from $\operatorname{RFU}_{\mathrm{a}}=\left[Y_{\mathrm{a}, \mathrm{t}} /\right.$ $\left(\right.$ MaxUptake $\left._{\mathrm{a}} \times D\right)$ ], where $Y$ is the deuterium uptake for peptide $a$ at incubation time $t$, and $D$ is the percentage of deuterium in the final labelling solution.

Confidence intervals for differential HDX-MS ( $\triangle \mathrm{HDX}$ ) measurements of any individual time point were then determined according to Houde et al. ${ }^{51}$ using Deuteros software (v. 1.0) ${ }^{34}$. There was no correlation found between $\triangle H D X$ values and their standard deviations $\left(R^{2}=0.09\right)$. Only peptides which satisfied a $\triangle$ HDX confidence interval of $98 \%$ were considered significant. All $\triangle \mathrm{HDX}$ AcrB structure figures were generated from the data using Deuteros in-house source code/ software ${ }^{34}$ and $\mathrm{Pymol}^{52}$. All supporting data and meta-data are reported in the Source data file and Supplementary Data 1-4.

The mass spectrometry proteomics data have been deposited to the ProteomeXchange Consortium via the PRIDE partner repository with the dataset identifier PXD019047 [http://www.ebi.ac.uk/pride/archive/projects/PXD019047].

Bacterial susceptibility assays. Antimicrobial susceptibility testing of all wildtype and mutant strains in the absence or presence of PA $\beta \mathrm{N}$ was determined in triplicate by the broth microdilution (BMD) method and the standardized agar doubling-dilution method as recommended by the European Committee on Antimicrobial Susceptibility Testing (EUCAST). EUCAST guidelines were followed conforming to ISO 20776-1:2006 53,54. Antibiotics and EPIs were made up and used according to the manufacturer's instructions. E. coli ATCC 25922 was used as the control strain.

Fluorescence polarization. AcrB ligand binding was determined using fluorescence polarization (FP) assays as performed by Su et al..$^{39}$. An AcrB-PA $\beta \mathrm{N}$ protein complex stock was prepared; to ensure a loaded complex, AcrB and $150 \mu \mathrm{M}$ PA $\beta \mathrm{N}$ was incubated for $2 \mathrm{~h}$ at $25^{\circ} \mathrm{C}$ before titrating with $1.5 \mu \mathrm{M}$ CIP $\left(k_{\mathrm{D}}\right.$ of PA $\beta \mathrm{N}$ is $15.72 \pm 3.0 \mu \mathrm{M}$, as measured by surface plasmon resonance ${ }^{50}$ ). AcrB protein titration experiments were performed in ligand binding solution $(50 \mathrm{mM}$ sodium phosphate, $150 \mathrm{mM}$ sodium chloride, $10 \%$ (v/v) glycerol, $1.5 \mu \mathrm{M}$ CIP, $150 \mu \mathrm{M}$ $\mathrm{PA} \beta \mathrm{N}, 0.03 \%(\mathrm{w} / \mathrm{v}) \mathrm{DDM}, \mathrm{pH} 7.4)$. FP measurements were taken after incubation for 5 min for each corresponding protein concentration to ensure that the binding has reached equilibrium. Ligand binding data was fit to a hyperbola function ( $\mathrm{FP}=$ $\left(B_{\max }{ }^{*}[\right.$ protein $\left.]\right) /\left(k_{\mathrm{D}}+\right.$ [protein $\left.\left.]\right)\right)$ as performed previously by Su et al. ${ }^{39}$ using ORIGIN Ver. 7.5. (OriginLab Corporation, Northampton, MA, USA).

A FP competition assay was performed by titrating increasing concentrations of PA $\beta \mathrm{N}(0-1000 \mu \mathrm{M})$ to a AcrB-CIP preformed complex concentration adjudged from the binding data $(1.5 \mu \mathrm{M}$ CIP and $45 \mu \mathrm{M}$ AcrB $)-C I P$ and AcrB were maintained at the same concentration during all $\mathrm{PA} \beta \mathrm{N}$ titrations. FP measurements were taken after incubation for $5 \mathrm{~min}$ for each corresponding protein concentration to ensure that the binding has reached equilibrium.

Each data point was an average of 15 FP measurements and each titrations series was performed three times. The absorption spectra of PA $\beta N$ from 350 to 500 $\mathrm{nm}$ exhibited that $\mathrm{PA} \beta \mathrm{N}$ absorbs light at 350 and $370 \mathrm{~nm}$. The excitation wavelength of CIP at $415 \mathrm{~nm}$ does not excite PA $\beta \mathrm{N}$, therefore PA $\beta \mathrm{N}$ can be considered as a non-fluorescent ligand within these experiments. DDM detergent concentration was consistent to eliminate possible changes in polarization by drug-DDM micelle interactions.

Molecular docking. A blind docking campaign was first performed using Autodock Vina ${ }^{55}$. As done in Atzori et al. ${ }^{56}$, a rectangular search space of size $125 \AA \times$ $125 \AA \times 110 \AA$ enclosing the whole portion of the protein potentially exposed to ligands was adopted. The exhaustiveness parameter, related to the extent of the exploration within the search space, was set to $8192(\sim 1000$ times the default 8$)$ in order to improve the sampling of docking poses within the large box used $(\sim 64$ times the default $30 \AA \times 30 \AA \times 30 \AA$ ). Flexibility of both partners was considered indirectly, by employing multiple conformations in ensemble docking runs ${ }^{57}$. For both CIP and PA $\beta N, 10$ representative molecular conformations were obtained from $1 \mu$ s long molecular dynamics simulations of the compounds in the presence of explicit solvent ${ }^{58}$ (data available at www.dsf.unica.it/translocation/db). Namely, a cluster analysis of the trajectories of the ligands was performed as described in Malloci et al. ${ }^{58}$, setting the number of cluster representatives to 10 .

For the wildtype receptor $\left(\mathrm{AcrB} \mathrm{WT}^{\mathrm{T}}\right), 10 \mathrm{X}$-ray asymmetric high-resolution structures (with PDB IDs: 2GIF, 2DHH, 2J8S, 3W9I, 4DX5, 4DX7, 4U8V, 4U8Y, $4 \mathrm{U} 95,4 \mathrm{U} 96)$ were considered, most bearing a substrate bound to the transporter. For the G288D variant of AcrB (AcrB ${ }^{\mathrm{G} 288 \mathrm{D}}$ ), we also employed 10 structures, namely the homology models derived on top of the $\mathrm{AcrB}^{\mathrm{WT}} \mathrm{X}$-ray structures mentioned above. Regarding the homology modelling protocol, the sequence of the G288D variant was first generated by manually modifying the FASTA file of the corresponding amino acid sequence of E. coli AcrB retrieved from the Uniprot database (Uniprot Id: P31224). Next, 100 homology models were generated for 
each template with the Modeller $9.21^{59}$ software. The variable target function method was used to perform the optimization, and the best model (that is the one with the highest value of the MOLPDF function) was employed in docking calculations.

The ensemble docking campaign resulted in several hundred poses per ligand, most of which were located inside the DBP of the monomer in the T-state $\left(\mathrm{DBP}_{\mathrm{T}}\right)$, which is the putative binding site for the recognition of low molecular mass compounds such as those studied here ${ }^{11}$ (see Supplementary Table 1). Because most docking poses were concentrated in this region, we performed a second docking campaign using a grid of $30 \AA \times 30 \AA \times 30 \AA$ and centred at $\mathrm{DBP}_{\mathrm{T}}$. Next, we performed a cluster analysis of the docking poses using as a metric the heavy atoms Root Mean Square Deviation (RMSD) of the substrate (setting the cut-off to $3 \AA$ ), which returned respectively $11,9,15$, and 17 different poses for the

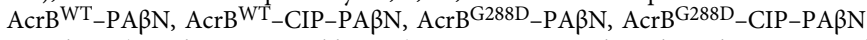
complexes (Supplementary Tables 2, 5). Moreover, to evaluate how the presence of $\mathrm{PA} \beta \mathrm{N}$ affects the binding of CIP in the ternary complexes, we selected three docking poses of CIP onto $\mathrm{AcrB}^{\mathrm{WT}}$ and $\mathrm{AcrB}^{\mathrm{G} 288 \mathrm{D}}$. In the case of the AcrB ${ }^{\mathrm{WT}}$-CIP complex, to consider the largest number of putative binding modes, we purposely selected docking poses with an orientation different than that reported previously ${ }^{29}$ (Supplementary Fig. 16).

Molecular dynamics simulations. All of the 52 complexes selected from docking runs were subjected to all-atom molecular dynamics (MD) simulations (each of 1 $\mu$ in length) performed with the AMBER18 package ${ }^{60}$.

Protomer-specific protonation states of AcrB were adopted following previous work ${ }^{61}$ : residues $\mathrm{E} 346$ and $\mathrm{D} 924$ were protonated only in the $\mathrm{L}$ and $\mathrm{T}$ protomers, while residues D407, D408, and D566 were protonated only in the O protomer, of AcrB. The topology and the initial coordinate files were created using the LEaP module of the AMBER18 package. The proteins were embedded in a mixed bilayer patch composed of 1-palmitoyl-2-oleoyl-sn-glycero-3-phosphoethanolamine (POPE) and 1-palmitoyl-2-oleoyl-sn-glycero-3-phosphoglycerol (POPG) in a 2/1 ratio, for a total of 660 lipid molecules symmetrically distributed in the two leaflets of the bilayer. The whole system was solvated with a $0.15 \mathrm{M}$ aqueous $\mathrm{NaCl}$ solution. The AMBER force-field protein.fb15 ${ }^{62}$ was used to represent the protein; lipid17 (http://ambermd. org/GetAmber.php) parameters were used for the POPE molecules; the TIP3PFB model was employed for water ${ }^{63}$. The General Amber Force-Field (GAFF) parameters ${ }^{64}$ for CIP and PA $\beta N$ were taken from Malloci et $\mathrm{a}^{58}$

Each system was first subjected to a multi-step structural relaxation via a combination of steepest descent and conjugate gradient methods using the pmemd program implemented in AMBER18, as described in previous publications ${ }^{4,29,61}$. The systems were then heated from 0 to $310 \mathrm{~K}$ in two subsequent MD simulations: (i) from 0 to $100 \mathrm{~K}$ in $1 \mathrm{~ns}$ under constant-volume conditions and with harmonic restraints $\left(k=1 \mathrm{kcal} \mathrm{mol}^{-1} \cdot \AA^{-2}\right)$ on the heavy atoms of both the protein and the lipids; (ii) from 100 to $310 \mathrm{~K}$ in $5 \mathrm{~ns}$ under constant pressure (set to a value of $1 \mathrm{~atm}$ ) and with restraints on the heavy atoms of the protein and on the $\mathrm{z}$ coordinates of the phosphorous atoms of the lipids to allow membrane rearrangement during heating. As a final equilibration step, a series of 20 equilibration steps, each of which was 500 ps in duration (total $10 \mathrm{~ns}$ ), with restraints on the protein coordinates, were performed to equilibrate the box dimensions. These equilibration steps were carried out under isotropic pressure scaling using the Berendsen barostat, whereas a Langevin thermostat (collision frequency of $1 \mathrm{ps}^{-1}$ ) was used to maintain a constant temperature. Finally, production MD simulations of $1 \mu \mathrm{s}$ were performed under an isothermal-isobaric ensemble for each system. A time step of $2 \mathrm{fs}$ was used for all runs before production, while the latter runs were carried out with a time step of $4 \mathrm{fs}$ after hydrogen mass repartitioning ${ }^{65}$.

During the MD simulations, the lengths of all the R-H bonds were constrained with the SHAKE algorithm. Coordinates were saved every 100 ps. The Particle mesh Ewald algorithm was used to evaluate long-range electrostatic forces with a non-bonded cut-off of $9 \AA$.

Post-processing of MD trajectories. MD trajectories were analyzed using either in-house $t c l$ and bash scripts or the cpptraj tool of AMBER18. Figures were prepared using gnuplot $5.0^{66}$ and VMD 1.9.367. All the calculations with the exception of the cluster analysis were performed on the conformations taken from the most populated conformational cluster (representing the most sampled conformation of the complex along the production trajectories) along the last $300 \mathrm{~ns}$ of the production runs.

Cluster analysis. Clustering of the ligand trajectory was carried out using the average-linkage hierarchical agglomerative clustering method implemented in cpptraj and employing an RMSD cut-off of $3 \AA$ calculated on all the heavy atoms of the ligand.

System stability. The RMSDs of the protein and of the substrates were calculated using cpptraj after structural alignment of each trajectory (reported in Supplementary Table 10). Namely, we calculated the Ca-RMSD of the protein with respect to the initial (docking) structure after alignment of the whole trimer. The RMSDs of the substrates were calculated with respect to the corresponding structure of the selected docking pose, as well as with respect to the last frame of the MD trajectory. In particular. To evaluate the magnitude of the displacements and reorientations of the substrates during the simulations, their RMSDs were calculated upon alignment of the $\mathrm{T}$ monomer of the protein to the reference frame.
Interaction network. Interactions stabilizing the complexes were analyzed by considering residues within $3.5 \AA$ of each substrate in the last $300 \mathrm{~ns}$ of the MD trajectories. Hydrogen bonds were identified through geometrical criteria, using a cut-off of $3.2 \AA$ for the distance between donor and acceptor atoms and a cut-off of $135^{\circ}$ for the donor-hydrogen-acceptor angle. Such analyses were conducted through in-house tcl scripts. Occupancy levels of hydrogen bonds and watermediated interactions (detected in the last $300 \mathrm{~ns}$ of each simulation) were also computed using cpptraj. For systems $\mathrm{AcrB}^{\mathrm{WT}}-\mathrm{PA} \beta \mathrm{N}, \mathrm{AcrB}^{\mathrm{WT}}$-CIP-PA $\beta \mathrm{N}$, $A c r B^{G 288 D}-P A \beta N$ and $A c r B{ }^{G 288 D}$-CIP-PA $\beta N$, the following analyses were also performed to evaluate their agreement with HDX-MS data.

System flexibility. The Root Mean Square Fluctuations (RMSFs) of the protein were calculated using cpptraj after structural alignment of each trajectory as described in the previous paragraph.

Hydration properties. Residue-wise average numbers of waters within the first (second) hydration layer were calculated with cpptraj using a distance cut-off of 3.4 (5.0) A between the nitrogen of the protein and the water oxygens.

Comparison with HDX-MS data. RMSFs and hydration properties of each system were compared with a proper reference state according to the current knowledge about the most likely conformations assumed by AcrB in the absence of ligands or complexed with substrates and inhibitors ${ }^{16}$. For instance, to account for conformational changes of AcrB induced by inhibitor binding, PA $\beta \mathrm{N}$-bound and apo AcrB structures were considered in their T- and L-state, respectively. The T-state was also considered for systems containing both $\mathrm{PA} \beta \mathrm{N}$ and $\mathrm{CIP}\left(\mathrm{AcrB}^{\mathrm{WT}}-\mathrm{CIP}-\mathrm{PA} \beta \mathrm{N}\right.$ and $\left.\mathrm{AcrB}{ }^{\mathrm{G} 288 \mathrm{D}}-\mathrm{CIP}-\mathrm{PA} \beta \mathrm{N}\right)$, hypothesizing their stability in this conformation, as evidenced by the RMSDs analyses conducted on our trajectories (Supplementary Figs. 6, 9, 12, 14). The list of reference states used for each analysis are reported in Supplementary Table 11.

Reporting summary. Further information on research design is available in the Nature Research Reporting Summary linked to this article.

\section{Data availability}

Data supporting the findings of this manuscript are available from the corresponding authors upon reasonable request. The molecular dynamics trajectories will be available anyone at any time by sending an e-mail to Attilio Vittorio Vargiu (vargiu@dsf.unica.it) A reporting summary for this Article is available as a Supplementary Information file. Mass spectrometry data files including processed DynamX files have been deposited to the ProteomeXchange Consortium via the PRIDE partner repository with the dataset identifier PXD019047. HDX-MS meta-data have been provided in Supplementary Data files 1-4 with this paper. Source data are provided with this paper.

Received: 29 April 2020; Accepted: 8 October 2020; Published online: 04 November 2020

\section{References}

1. Du, D. et al. Multidrug efflux pumps: structure, function and regulation. Nat. Rev. Microbiol. 16, 523-539 (2018).

2. Du, D. et al. Structure of the AcrAB-TolC multidrug efflux pump. Nature 509, 512-515 (2014).

3. Symmons, M. F., Bokma, E., Koronakis, E., Hughes, C. \& Koronakis, V. The assembled structure of a complete tripartite bacterial multidrug efflux pump. Proc. Natl Acad. Sci. U.S.A. 106, 7173-7178 (2009).

4. Sjuts, H. et al. Molecular basis for inhibition of AcrB multidrug efflux pump by novel and powerful pyranopyridine derivatives. Proc. Natl Acad. Sci. U.S.A 113, 3509-3514 (2016).

5. Kinana, A. D., Vargiu, A. V., May, T. \& Nikaido, H. Aminoacyl $\beta$ naphthylamides as substrates and modulators of AcrB multidrug efflux pump. Proc. Natl Acad. Sci. U.S.A. 113, 1405-1410 (2016).

6. Vargiu, A. V., Ruggerone, P., Opperman, T. J., Nguyen, S. T. \& Nikaido, H. Molecular mechanism of MBX2319 inhibition of Escherichia coli AcrB multidrug efflux pump and comparison with other inhibitors. Antimicrob. Agents Chemother. 58, 6224-6234 (2014).

7. Yu, E. W., McDermott, G., Zgurskaya, H. I., Nikaido, H. \& Koshland, D. E. Jr. Structural basis of multiple drug-binding capacity of the AcrB multidrug efflux pump. Science 300, 976-980 (2003)

8. Zwama, M. \& Yamaguchi, A. Molecular mechanisms of AcrB-mediated multidrug export. Res. Microbiol. 169, 372-383 (2018).

9. Murakami, S., Nakashima, R., Yamashita, E., Matsumoto, T. \& Yamaguchi, A. Crystal structures of a multidrug transporter reveal a functionally rotating mechanism. Nature 443, 173-179 (2006).

10. Eicher, T. et al. Transport of drugs by the multidrug transporter AcrB involves an access and a deep binding pocket that are separated by a switch-loop. Proc. Natl Acad. Sci. U.S.A. 109, 5687-5692 (2012). 
11. Nakashima, R., Sakurai, K., Yamasaki, S., Nishino, K. \& Yamaguchi, A. Structures of the multidrug exporter AcrB reveal a proximal multisite drugbinding pocket. Nature 480, 565-569 (2011).

12. Seeger, M. A. et al. Structural asymmetry of AcrB trimer suggests a peristaltic pump mechanism. Science 313, 1295-1298 (2006).

13. Murakami, S., Nakashima, R., Yamashita, E. \& Yamaguchi, A. Crystal structure of bacterial multidrug efflux transporter AcrB. Nature 419, 587-593 (2002).

14. Eicher, T. et al. Coupling of remote alternating-access transport mechanisms for protons and substrates in the multidrug efflux pump AcrB. Elife 3, e03145 (2014).

15. Yu, E. W., Aires, J. R., McDermott, G. \& Nikaido, H. A periplasmic drugbinding site of the AcrB multidrug efflux pump: a crystallographic and sitedirected mutagenesis study. J. Bacteriol. 187, 6804-6815 (2005).

16. Wang, Z. et al. An allosteric transport mechanism for the AcrAB-TolC multidrug efflux pump. eLife 6, e24905 (2017).

17. Ruggerone, P., Murakami, S., Pos, K. M. \& Vargiu, A. V. RND efflux pumps: structural information translated into function and inhibition mechanisms. Curr. Top. Med. Chem. 13, 3079-3100 (2013).

18. Oswald, C., Tam, H.-K. \& Pos, K. M. Transport of lipophilic carboxylates is mediated by transmembrane helix 2 in multidrug transporter AcrB. Nat. Commun. 7, 13819 (2016).

19. Matsunaga, Y. et al. Energetics and conformational pathways of functional rotation in the multidrug transporter AcrB. eLife 7, e31715 (2018).

20. Vargiu, A. V. et al. Water-mediated interactions enable smooth substrate transport in a bacterial efflux pump. Biochim. Biophys. Acta, Gen. Subj. 1862, 836-845 (2018).

21. Muller, R. T. et al. Switch loop flexibility affects substrate transport of the AcrB efflux pump. J. Mol. Biol. 429, 3863-3874 (2017).

22. Ababou, A. \& Koronakis, V. Structures of gate loop variants of the AcrB drug efflux pump bound by erythromycin substrate. PLoS ONE 11, e0159154 (2016).

23. Fang, J., Yu, L., Wu, M. \& Wei, Y. Dissecting the function of a protruding loop in AcrB trimerization. J. Biomol. Struct. Dyn. 31, 385-392 (2013).

24. Zwama, M. et al. Hoisting-loop in bacterial multidrug exporter AcrB is a highly flexible hinge that enables the large motion of the subdomains. Front. Microbiol. 8, 2095-2095 (2017).

25. Karplus, M. \& Kuriyan, J. Molecular dynamics and protein function. Proc. Natl Acad. Sci. U.S.A. 102, 6679-6685 (2005).

26. Shaw, D. E. et al. Atomic-level characterization of the structural dynamics of proteins. Science 330, 341-346 (2010).

27. Bhabha, G. et al. A dynamic knockout reveals that conformational fluctuations influence the chemical step of enzyme catalysis. Science 332, 234-238 (2011).

28. Campbell, E. et al. The role of protein dynamics in the evolution of new enzyme function. Nat. Chem. Biol. 12, 944-950 (2016).

29. Vargiu, A. V. \& Nikaido, H. Multidrug binding properties of the AcrB efflux pump characterized by molecular dynamics simulations. Proc. Natl Acad. Sci. U.S.A. 109, 20637-20642 (2012).

30. Blair, J. M. A. et al. AcrB drug-binding pocket substitution confers clinically relevant resistance and altered substrate specificity. Proc. Natl Acad. Sci. U.S.A. 112, 3511-3516 (2015)

31. Trabjerg, E., Nazari, Z. E. \& Rand, K. D. Conformational analysis of complex protein states by hydrogen/deuterium exchange mass spectrometry (HDX-MS): Challenges and emerging solutions. Trends Anal. Chem. 106, 125-138 (2018).

32. Wales, T. E., Eggertson, M. J. \& Engen, J. R. Considerations in the analysis of hydrogen exchange mass spectrometry data. Methods Mol. Biol. 1007, 263-288 (2013).

33. Masson, G. R. et al. Recommendations for performing, interpreting and reporting hydrogen deuterium exchange mass spectrometry (HDX-MS) experiments. Nat. Methods 16, 595-602 (2019).

34. Lau, A. M. C., Ahdash, Z., Martens, C. \& Politis, A. Deuteros: software for rapid analysis and visualization of data from differential hydrogen deuterium exchange-mass spectrometry. Bioinformatics 35, 3171-3173 (2019).

35. Chalmers, M. J., Busby, S. A., Pascal, B. D., West, G. M. \& Griffin, P. R. Differential hydrogen/deuterium exchange mass spectrometry analysis of protein-ligand interactions. Expert Rev. Proteomics 8, 43-59 (2011).

36. Nakashima, R. et al. Structural basis for the inhibition of bacterial multidrug exporters. Nature 500, 102-106 (2013).

37. Petruk, A. A. et al. Molecular dynamics simulations provide atomistic insight into hydrogen exchange mass spectrometry experiments. J. Chem. Theory Comput. 9, 658-669 (2013).

38. Tyers, M. \& Wright, G. D. Drug combinations: a strategy to extend the life of antibiotics in the 21st century. Nat. Rev. Microbiol. 17, 141-155 (2019).

39. Su, C. C. \& Yu, E. W. Ligand-transporter interaction in the AcrB multidrug efflux pump determined by fluorescence polarization assay. FEBS Lett. 581, 4972-4976 (2007).

40. Laganowsky, A. et al. Membrane proteins bind lipids selectively to modulate their structure and function. Nature 510, 172-175 (2014)
41. Qiu, W. et al. Structure and activity of lipid bilayer within a membraneprotein transporter. Proc. Natl Acad. Sci. U.S.A. 115, 12985-12990 (2018).

42. Johnson, R. M. et al. Cryo-EM structure and molecular dynamics analysis of the fluoroquinolone resistant mutant of the AcrB transporter from Salmonella. Microorg. 8, 943 (2020).

43. Yao, X., Fan, X. \& Yan, N. Cryo-EM analysis of a membrane protein embedded in the liposome. Proc. Natl Acad. Sci. U.S.A. 117, 18497-18503 (2020).

44. Reading, E. et al. The role of the detergent micelle in preserving the structure of membrane proteins in the gas phase. Angew. Chem. Int. Ed. Engl. 54, 4577-4581 (2015)

45. Reading, E. et al. The effect of detergent, temperature, and lipid on the oligomeric state of MscL constructs: insights from mass spectrometry. Chem. Biol. 22, 593-603 (2015).

46. Gasteiger, E. et al. in The Proteomics Protocols Handbook (ed Walker, J. M.) 571-607 (Humana Press, 2005).

47. Lees, J. G., Smith, B. R., Wien, F., Miles, A. J. \& Wallace, B. A. CDtool-an integrated software package for circular dichroism spectroscopic data processing, analysis, and archiving. Anal. Biochem. 332, 285-289 (2004).

48. Laganowsky, A., Reading, E., Hopper, J. T. \& Robinson, C. V. Mass spectrometry of intact membrane protein complexes. Nat. Protoc. 8, 639-651 (2013).

49. Chandramohan, A. et al. Predicting allosteric effects from orthosteric binding in Hsp90-Ligand interactions: implications for fragment-based drug design. PLOS Comput. Biol. 12, e1004840 (2016).

50. Mowla, R., Wang, Y., Ma, S. \& Venter, H. Kinetic analysis of the inhibition of the drug efflux protein AcrB using surface plasmon resonance. Biochim. Biophys. Acta, Biomembr. 1860, 878-886 (2018).

51. Houde, D., Berkowitz, S. A. \& Engen, J. R. The utility of hydrogen/deuterium exchange mass spectrometry in biopharmaceutical comparability studies. $J$. Pharm. Sci. 100, 2071-2086 (2011).

52. Schrodinger, L. L. C. The PyMOL Molecular Graphics System Version 1.3r1 https://pymol.org/2/support.html? (2010).

53. EUCAST Definitive Document E.DEF 3.1, June 2000: Determination of minimum inhibitory concentrations (MICs) of antibacterial agents by agar dilution. Clin. Microbiol. Infect. 6, 509-515 (2000).

54. ISO. Clinical Laboratory Testing and In Vitro Diagnostic Test SystemsSusceptibility Testing of Infectious Agents and Evaluation of Performance of Antimicrobial Susceptibility Test Devices-Part 1: Reference Method for Testing the In Vitro Activity of Antimicrobial Agents Against Rapidly Growing Aerobic Bacteria Involved in Infectious Diseases (International Organization for Standardization, Geneva, Switzerland, 2006).

55. Trott, O. \& Olson, A. J. AutoDock Vina: improving the speed and accuracy of docking with a new scoring function, efficient optimization, and multithreading. J. Comput. Chem. 31, 455-461 (2010).

56. Atzori, A. et al. Identification and characterization of carbapenem binding sites within the RND-transporter AcrB. Biochim. Biophys. Acta, Biomembr. 1861, 62-74 (2019).

57. Amaro, R. E. et al. Ensemble docking in drug discovery. Biophys. J. 114 2271-2278 (2018).

58. Malloci, G. et al. A database of force-field parameters, dynamics, and properties of antimicrobial compounds. Molecules 20, 13997-14021 (2015).

59. Šali, A. \& Blundell, T. L. Comparative protein modelling by satisfaction of spatial restraints. J. Mol. Biol. 234, 779-815 (1993).

60. Case, D. A. et al. AMBER 2018. (University of California, San Francisco, 2018) https://ambermd.org/CiteAmber.php.

61. Ramaswamy, V. K., Vargiu, A. V., Malloci, G., Dreier, J. \& Ruggerone, P. Molecular rationale behind the differential substrate specificity of bacterial RND multi-drug transporters. Sci. Rep. 7, 8075 (2017).

62. Wang, L.-P. et al. Building a more predictive protein force field: a systematic and reproducible route to AMBER-FB15. J. Phys. Chem. B 121, 4023-4039 (2017).

63. Gonzalez-Salgado, D. \& Vega, C. A new intermolecular potential for simulations of methanol: The OPLS/2016 model. J. Chem. Phys. 145, 034508 (2016).

64. Wang, J., Wolf, R. M., Caldwell, J. W., Kollman, P. A. \& Case, D. A. Development and testing of a general amber force field. J. Comput. Chem. 25 , 1157-1174 (2004)

65. Hopkins, C. W., Le Grand, S., Walker, R. C. \& Roitberg, A. E. Long-time-step molecular dynamics through hydrogen mass repartitioning. J. Chem. Theory Comput. 11, 1864-1874 (2015).

66. Williams, T. \& Kelley, C. Gnuplot 5.0: an interactive plotting program. (2017) http://gnuplot.info

67. Humphrey, W., Dalke, A. \& Schulten, K. VMD: visual molecular dynamics. J. Mol. Graph 14, 33-38 (1996).

68. Jurcik, A. et al. CAVER Analyst 2.0: analysis and visualization of channels and tunnels in protein structures and molecular dynamics trajectories. Bioinformatics 34, 3586-3588 (2018). 


\section{Acknowledgements}

Work at King's College London was supported by a UKRI Future Leaders Fellowship (MR/S015426/1) to E.R., the Wellcome Trust (109854/Z/15/Z) to A.P., a Wellcome Trust Investigator Award 214259 to P.J.B., and a King's Health Partners R\&D Challenge Fund through the MRC Confidence in Concept Grant (MC_PC_15031) to A.P., E.R., and P.J.B. Work at the University of Cagliari received support from the National Institutes of Allergy and Infectious Diseases project number AI136799 (C.F., G.M., P.R., and A.V.V.) and the Innovative Medicines Initiatives Joint Undertaking under grant agreement number 115525 (C.F., G.M., P.R., and A.V.V.), resources that are composed of financial contribution from the European Union 7th Framework Programme (FP7/2007-2013) and EFPIA companies in kind contribution. Work at the University of Birmingham by L.J.V.P., X.W.K., J.S., and V.R. was supported by MRC grant, (MR/P022596/1) and E.M. G. by an MRC iCASE studentship (MR/N017846/1). We thank Bram Snijders, Peter Faull and Shahid Mehmood (Francis Crick Institute, London) for the use of their Thermo Scientific Q Exactive UHMR hybrid Quadrupole-orbitrap mass spectrometer, and Andrea Bosin and Giovanni Serra (University of Cagliari) for technical assistance with the use of HPC resources. We thank H. Zgurskaya and O. Lomovskaya for enlightening discussions during the revision of the manuscript.

\section{Author contributions}

E.R., Z.A., A.V.V, L.J.V.P., and A.P. designed the research. E.R., Z.A., A.M.L., and H.F. performed all experiments and analyses, except for molecular modelling and bacterial susceptibility assays. C.F., G.M., and A.V.V. carried out docking, molecular dynamics and performed post-molecular dynamics analyses. X.W.K., V.R., J.S., and E.M.G. performed bacterial susceptibility assays and analysis. A.K. and Z.A. performed UHMR Native MS measurements and analysis. E.R., P.J.B., P.R., A.V.V, L.J.V.P., and A.P. supervised the project. E.R., Z.A., and A.P. wrote the manuscript with input from the other authors.

\section{Competing interests}

The authors declare no competing interests.

\section{Additional information}

Supplementary information is available for this paper at https://doi.org/10.1038/s41467020-19397-2.

Correspondence and requests for materials should be addressed to E.R. or A.P.

Reprints and permission information is available at http://www.nature.com/reprints

Publisher's note Springer Nature remains neutral with regard to jurisdictional claims in published maps and institutional affiliations.

cc) Open Access This article is licensed under a Creative Commons Attribution 4.0 International License, which permits use, sharing, adaptation, distribution and reproduction in any medium or format, as long as you give appropriate credit to the original author(s) and the source, provide a link to the Creative Commons license, and indicate if changes were made. The images or other third party material in this article are included in the article's Creative Commons license, unless indicated otherwise in a credit line to the material. If material is not included in the article's Creative Commons license and your intended use is not permitted by statutory regulation or exceeds the permitted use, you will need to obtain permission directly from the copyright holder. To view a copy of this license, visit http://creativecommons.org/ licenses/by/4.0/.

(C) The Author(s) 2020 